\title{
Opportunistic Distributed Space-Time Coding for Decode-and-Forward Cooperation Systems
}

\author{
Yulong Zou, Member, IEEE, Yu-Dong Yao, Fellow, IEEE, and Baoyu Zheng, Member, IEEE
}

\begin{abstract}
In this paper, we consider a decode-and-forward (DF) cooperation system consisting of two cooperative users in sending their information to a common destination, for which the distributed space-time coding (DSTC) is applied in an opportunistic manner, called opportunistic DSTC (O-DSTC), depending on whether the two users succeed in decoding each other's information or not. We propose two O-DSTC schemes for the full-duplex and half-duplex relaying scenarios, which are, respectively, referred to as the full-duplex and half-duplex-based O-DSTC. We evaluate the outage performance of the proposed O-DSTC as well as the conventional selective DF (S-DF) cooperation and fixed DSTC (F-DSTC) schemes. Numerical results show that the O-DSTC outperforms the conventional S-DF and F-DSTC schemes considering both full-duplex and half-duplex. In addition, we develop the diversity-multiplexing tradeoff (DMT) of the proposed O-DSTC, conventional S-DF and F-DSTC schemes by considering the two cooperative users with different data rates (also known as different multiplexing gains). We show that, for both the full-duplex and half-duplex modes, the proposed O-DSTC strictly outperforms the conventional S-DF and F-DSTC in terms of DMT. It is also shown that, in the full-duplex-based O-DSTC scheme, the diversity gain obtained by any of the two cooperative users not only depends on its own multiplexing gain, but also relates to its partner's multiplexing gain. By jointly considering the two users' DMT, the full-duplex-based O-DSTC scheme can achieve the optimal diversity gain when the two users are with the same multiplexing gain. For the half-duplex-based O-DSTC scheme, the DMT performance of the two users are independent of each other, which differs from the full-duplex-based O-DSTC scheme where mutual dependence exists between the cooperative users in terms of DMT.
\end{abstract}

Index Terms-Cooperative diversity, decode-and-forward (DF), distributed space-time coding (DSTC), diversity-multiplexing tradeoff (DMT), outage probability.

Manuscript received May 22, 2011; revised September 19, 2011; accepted December 12, 2011. Date of publication December 23, 2011; date of current version March 06, 2012. The associate editor coordinating the review of this manuscript and approving it for publication was Prof. David Love. This work was partially supported by the Postgraduate Innovation Program of Scientific Research of Jiangsu Province (Grant Nos. CX08B 080Z, CX09B 150Z), the Key Project of Nature Science Funding of Jiangsu Province (Grant No. BK2010077), and the National Natural Science Foundation of China (Grant No. 60972039).

Y. Zou is with the Institute of Signal Processing and Transmission, Nanjing University of Posts and Telecommunications, Nanjing, Jiangsu 210003, China. He is also with the Electrical and Computer Engineering Department, Stevens Institute of Technology, Hoboken, NJ 07030 USA (e-mail: zouyulong198412@126.com; yzou1@stevens.edu).

Y.-D. Yao is with the Electrical and Computer Engineering Department, Stevens Institute of Technology, Hoboken, NJ 07030, USA (e-mail: yyao@stevens.edu).

B. Zheng is with the Institute of Signal Processing and Transmission, Nanjing University of Posts and Telecommunications, Nanjing, Jiangsu 210003, China (e-mail: zby@njupt.edu.cn).

Color versions of one or more of the figures in this paper are available online at http://ieeexplore.ieee.org.

Digital Object Identifier 10.1109/TSP.2011.2181503

\section{INTRODUCTION}

A $\mathrm{S}$ is well known, transmit diversity has been proposed by sending signal through multiple antennas to combat fading effects, which is widely recognized as an effective means to improve wireless transmission performance [1], e.g., Alamouti $2 \times 1$ space-time coding has been incorporated into various cellular and wireless standards. However, in some practical scenarios (e.g., handheld terminals, sensor nodes, etc.), it may be difficult to support multiple antennas due to the terminal size, power consumption, and hardware limitations [2]. To that end, cooperative diversity is emerging as an alternative method to obtain the transmit diversity by allowing single-antenna terminals to share their antennas to form a virtual antenna array [3]. Recently, cooperative diversity has been studied extensively from different perspectives, e.g., outage probability analysis [4], [5], diversity-multiplexing tradeoff (DMT) [6]-[9], and its applications to emerging cognitive radio networks [10]-[12].

\section{A. Related Works}

Cooperative diversity has been first introduced in [3] by considering two cooperative users in a code-division multiple access (CDMA) scenario, where achievable data rate regions for cooperative users are developed. Then, in [4], the authors have studied two users in assisting each other's transmissions with a half-duplex relay regime and examined several relaying protocols. It has been shown in [4] that the diversity gain achieved typically comes at the expense of multiplexing gain, since two channels are required for each message transmission from source via relay to destination. To alleviate the multiplexing gain loss, a dynamic decode-and-forward (DDF) strategy has been proposed in [6] by allowing both source and relay nodes to transmit their independent Gaussian codewords in the same channel, for which a DMT analysis is conducted in an information-theoretic sense. Besides, in [7] and [8], the authors have examined a superposition coding approach to improve the multiplexing gain of cooperative networks, where each user transmits a linear combination of its own information and the others' information. The superposition decoding typically uses log-likelihood ratios to extract multiple modulated symbols from one superposition codeword, which, however, requires precise instantaneous fading gains and noise variance, in addition to linear combination coefficients that the superposition codeword employs. Moreover, as mentioned in [7], improper combination coefficients would make the superposition coding approach break down. However, how to perform an efficient design for such coefficients is unknown and 
very challenging, especially for multiple users with high-order modulation.

As an alternative, space-time coding has been shown as an effective method in traditional multiple-antenna systems to increase the reliability and capacity of a wireless link [13]-[15]. Hence, it is of interest to utilize space-time coding for cooperative diversity, which is called distributed space-time coding (DSTC) since its encoding process is operated among distributed cooperative users' antennas. DSTC has been first explored for the cooperative diversity in [16], where each message transmission from a source to its destination requires two phases: 1) the source multicasts its message to the cooperative terminals and destination, and 2) these terminals (that successfully decode the source message) retransmit a space-time coded version of their decoded results. In [17], the authors have studied the application of space-time coding to an amplify-and-forward relay network and proposed a two-step cooperative relaying protocol. To be specific, in the first step, a source transmits a message and, in the next step, relay nodes encode their received signals into a "distributed" linear dispersion code, and forward the coded signals to destination. In [18], the authors have investigated the Alamouti space-time coding for regenerative relay networks and analyzed the effect of intermediate decision errors at a cooperative relay, where the relay first decodes its received signals, and then re-encodes (based on the Alamouti coding) and forwards the decoded outcomes to the destination. More recently, [19] has examined the use of Alamouti space-time coding in a nonregenerative relay network by allowing each relay to retransmit an appropriately scaled Alamouti coded version of its received signal, where the scaling factor is adapted to channel conditions to minimize the outage probability.

\section{B. Motivation and Contribution}

It is important to note that the performance improvement achieved by all previous DSTC researches [16]-[19] in terms of diversity gain comes at the cost of one-half of multiplexing gain. In this paper, we investigate opportunistic distributed spacetime coding (O-DSTC) with full-diversity and, at the same time, with an increased multiplexing gain as compared to [4] and [16]-[19]. We first consider the full-duplex relay regime and propose a full-duplex-based O-DSTC scheme, which is proven as a full-diversity and full-rate code. We then consider the halfduplex relay scenario and propose a half-duplex-based O-DSTC scheme. We show that the half-duplex-based O-DSTC scheme achieves full diversity and a maximum multiplexing gain of twothird. This is attractive as compared with previous researches [4], [16]-[19] where a maximum multiplexing gain of only onehalf is obtained. The main contributions of this paper are described as follows.

- We consider a decode-and-forward cooperation system that consists of two users assisting each other in sending information to a common destination. Note that the two-user cooperation is an essential scenario to be addressed, since a general multiple-user scenario can typically be converted to a two-user cooperation by designing an additional grouping and partner selection protocol [21].
We explore so-called opportunistic distributed space-time coding (O-DSTC) and propose two O-DSTC schemes for the full-duplex and half-duplex regimes, called the full-duplex and half-duplex-based O-DSTC, respectively. It is worth mentioning that a fully distributed approach is proposed to implement the half-duplex-based O-DSTC scheme without any feedback information between any two network nodes (including the two cooperative users and destination).

- We derive closed-form outage probability expressions of the proposed full-duplex and half-duplex-based O-DSTC schemes as well as the conventional selective decode-andforward (S-DF) cooperation [4] and the fixed distributed space-time coding (F-DSTC) [18], [19] where the Alamouti space-time coding [13] is always applied. We illustrate the advantage of proposed O-DSTC over the conventional S-DF and F-DSTC schemes in terms of the outage probability. It is pointed out that the proposed O-DSTC outperforms the conventional S-DF cooperation by about $3-5 \mathrm{~dB}$, which indirectly shows its advantage over the superposition modulation-based cooperative diversity [7], [8], since the latter approach outperforms the conventional S-DF by $1.5-2 \mathrm{~dB}$ only as reported in [7] and [8].

- We examine the DMT performance of the full-duplex and half-duplex-based O-DSTC and F-DSTC schemes by considering the two cooperative users with different data rates. It is shown that, no matter which duplex mode is used, the O-DSTC strictly outperforms the conventional S-DF cooperation [4] and F-DSTC [18] in terms of DMT. We show that, in the full-duplex-based O-DSTC scheme, the diversity gain achieved by any of the two cooperative users depends on both the two users' multiplexing gains and by jointly considering the two users' DMT, the optimal diversity gain can be obtained when the two users are with the same multiplexing gain. For the half-duplex-based O-DSTC scheme, the diversity gain of a user only depends on its own multiplexing gain and the DMT performance of the two users are independent of each other.

The remainder of this paper is organized as follows. After a brief description of the system model in Section II, we propose the full-duplex and half-duplex-based O-DSTC schemes, followed by Section III, where an outage analysis of the proposed O-DSTC, traditional F-DSTC [18] and S-DF cooperation [4] is presented along with the numerical outage probability results. Next, in Section IV, we investigate the DMT performance of the full-duplex and half-duplex-based O-DSTC and F-DSTC schemes. Finally, in Section V, we make some concluding remarks.

\section{Proposed Opportunistic Distributed Space-Time CODING (O-DSTC) SCHEMES}

In this section, we first present the system model used throughout this paper. Next, we propose the O-DSTC schemes with full-duplex and half-duplex considerations, which are referred to as the full-duplex and half-duplex-based O-DSTC, respectively. For the comparison purpose, we also present the conventional S-DF cooperation as proposed in [4]. 


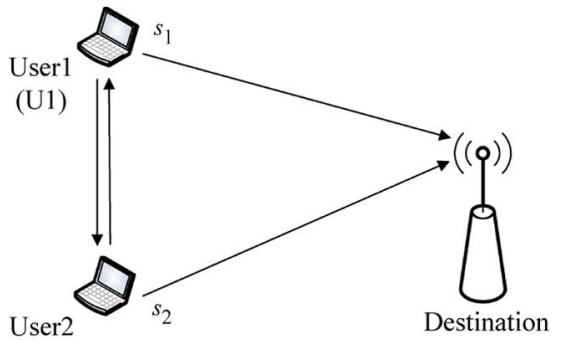

(U2)

Fig. 1. A decode-and-forward cooperation system with two cooperative users transmitting data to a common destination.

\section{A. System Model}

As shown in Fig. 1, we consider a cooperative diversity system consisting of two cooperative users (as denoted by $\mathrm{U} 1$ and U2), which assist each other using a DF protocol in transmitting their information (i.e., $s_{1}$ and $s_{2}$ ) to a common destination, where the subscripts 1 and 2 represent $\mathrm{U} 1$ and $\mathrm{U} 2$, respectively. Although only two cooperative users are considered in this paper, this is an essential scenario to be addressed, since a more generic scenario with multiple source users can be typically converted to the two-user cooperation by designing an additional grouping and partner selection protocol [21]. In addition, each node as shown in Fig. 1 is assumed to have a single antenna, for which two duplex modes (i.e., full-duplex and half-duplex) are considered in the paper. It is pointed out that full-duplex and half-duplex [20] refer to the antenna with and without the capability of transmitting and receiving a signal simultaneously over the same channel, respectively.

Fig. 2(a) illustrates that the proposed full-duplex-based O-DSTC scheme divides a total block into two time frames which are shared between $\mathrm{U} 1$ and $\mathrm{U} 2$. In the first time frame, both $\mathrm{U} 1$ and $\mathrm{U} 2$, respectively, transmit their own information $s_{1}$ and $s_{2}$ to the destination. At the same time, by considering the full-duplex regime, $\mathrm{U} 1$ and $\mathrm{U} 2$ can receive and decode each other's information over the channels between the two users, called inter-user channels. In the subsequent time frame, $\mathrm{U} 1$ and $\mathrm{U} 2$ transmit $s_{1}$ and $s_{2}$ in an opportunistic encoding manner depending on whether U1 and U2 decode each other's information successfully or not, which will be discussed in details in Section II-B. One can observe from Fig. 2(a) that the full-duplex-based O-DSTC utilizes two time frames for transmitting two symbols (i.e., $s_{1}$ and $s_{2}$ ), implying that full multiplexing gain (also known as full rate) is achieved. We will prove in Section IV that such an O-DSTC scheme also achieves the full diversity gain and, in contrast, the traditional fixed DSTC (F-DSTC) is unable to achieve full diversity due to the bottleneck effect caused by inter-user channels. Notice that, in the traditional F-DSTC scheme, either U1 or U2 failing to decoding its partner's information will result in interference at the destination in decoding both the two users' information, as will be shown in (44).

In Fig. 2(b), we depict the half-duplex-based O-DSTC, where a total block is divided into three time frames. The difference between the full-duplex and half-duplex-based O-DSTC is that the former scheme utilizes one time frame to exchange the information between $\mathrm{U} 1$ and $\mathrm{U} 2$, however the half-duplex-based

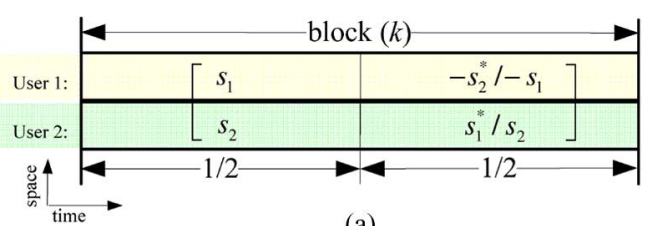

(a)

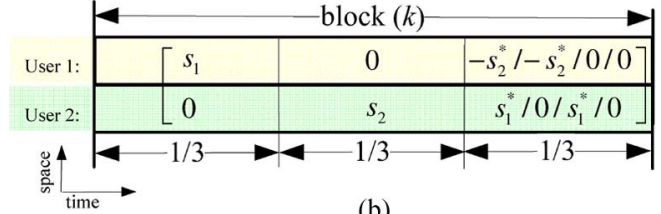

(b)

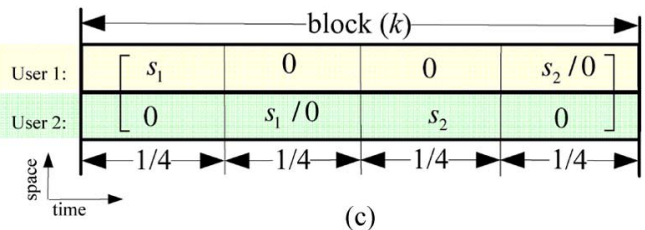

(c)

Fig. 2. Opportunistic distributed space-time coding (O-DSTC) and conventional S-DF [4] structures. (a) Full-duplex-based O-DSTC. (b) Half-duplexbased O-DSTC. (c) Conventional S-DF cooperation.

O-DSTC requires two frames to complete the exchange process. From Fig. 2(b), one can see that, in the first two time frames, $\mathrm{U} 1$ and $\mathrm{U} 2$, respectively, broadcast $s_{1}$ and $s_{2}$ to each other and the destination. During the third time frame, U1 and U2 encode and transmit $s_{1}$ and $s_{2}$ using an opportunistic encoding approach, for which a detailed explanation will be presented in Section II-C. As shown in Fig. 2(b), the half-duplex-based O-DSTC uses three time frames for the transmission of $s_{1}$ and $s_{2}$, thus a maximum multiplexing gain of two-third is achieved. This is very attractive as compared with the conventional S-DF cooperation [4] and previous DSTC researches [16]-[19] where a maximum multiplexing gain of only one-half is obtained.

Fig. 2(c) shows the conventional S-DF cooperation scheme as proposed in [4], where U1 and U2 are assisting each other's data transmissions (i.e., $s_{1}$ and $s_{2}$ ) using four time frames. Specifically, in the first time frame of block $k$, $\mathrm{U} 1$ broadcasts its own information $s_{1}$ to the destination and $\mathrm{U} 2$ that attempts to decode its received signal. Then, in the second time frame, U2 forwards its decoded outcome in a selective manner depending on whether it succeeds in decoding $s_{1}$ or not. If U2 decodes U1's transmission $\left(s_{1}\right)$ successfully, it will forward $s_{1}$ to the destination. Otherwise, U2 just keeps silent in the second time frame. The process of transmitting $s_{2}$ during the remaining two time frames of block $k$ is essentially same as the procedure of transmitting $s_{1}$ in the first two frames. One can see from Fig. 2(c) that four time frames are used to complete the transmissions of $s_{1}$ and $s_{2}$, implying that a maximum multiplexing gain of one-half only is achieved by the conventional S-DF cooperation [4].

In addition, each channel between any two nodes as shown in Fig. 1 is modeled as Rayleigh block fading, which is constant during one time block and varies independently in the next time block. Assume that all channels are independent of each other and the channel state information (CSI) is available at a receiver. Moreover, the receiver has additive white Gaussian noise (AWGN) with zero mean and variance $N_{0}$. 


\section{B. Full-Duplex-Based O-DSTC Scheme}

As a baseline, let us consider the noncooperative transmission with one block consisting of two time frames where two users take turns in accessing the time frames to transmit their own data with power $E_{s}$ at data rates $R_{1}$ and $R_{2}$ in bits per frame, respectively. One can see from Fig. 2(a) that, in the full-duplex-based O-DSTC, two independent symbols $s_{1}$ and $s_{2}$ are transmitted by using two time frames, which means that no extra channel resource is wasted by retransmission. Thus, when U1 and $\mathrm{U} 2$, respectively, transmit at data rates $R_{1}$ and $R_{2}$ in the full-duplex-based O-DSTC scheme, it is guaranteed to transmit the same amount information (during one block) as the noncooperative scheme. However, the proposed full-duplex-based O-DSTC scheme requires both $\mathrm{U} 1$ and $\mathrm{U} 2$ always transmitting in two frames during one block, differing from noncooperative scheme where U1 and U2 take turns in the time block to transmit their information. Hence, for a fair comparison with the noncooperative transmission in terms of power consumption, we consider one-half power $\frac{E_{s}}{2}$ for each user during one time frame in the full-duplex-based O-DSTC scheme. Accordingly, the received signal at the destination in the first time frame of block $k$ is expressed as

$$
y_{d}^{1}=\sqrt{\frac{E_{s}}{2}} h_{1 d} s_{1}+\sqrt{\frac{E_{s}}{2}} h_{2 d} s_{2}+n_{d}^{1}
$$

where the superscript 1 represents the first time frame of block $k, h_{1 d}$ and $h_{2 d}$ are fading coefficients of the channel from U1 to destination and that from $\mathrm{U} 2$ to destination, respectively, and $n_{d}^{1}$ represents AWGN with zero mean and variance $N_{0}$. Note that the fading coefficients are modeled as constant during one block (including two frames for full-duplex-based O-DSTC) and vary independently in next time block. Meanwhile, the full-duplex enables U1 and U2 to receive and decode each other's information over the interuser channels at the same time. Hence, the received signals at $\mathrm{U} 1$ and $\mathrm{U} 2$ are, respectively, given by

$$
y_{1}=\sqrt{\frac{E_{s}}{2}} h_{21} s_{2}+n_{1}
$$

where $h_{21}$ represents the channel from $\mathrm{U} 2$ to $\mathrm{U} 1$ and $n_{1}$ is AWGN with zero mean and variance $N_{0}$, and

$$
y_{2}=\sqrt{\frac{E_{s}}{2}} h_{12} s_{1}+n_{2}
$$

where $h_{12}$ represents the channel from $\mathrm{U} 1$ to $\mathrm{U} 2$ and $n_{2}$ is AWGN with zero mean and variance $N_{0}$. Then, U1 and U2 decode each other's information using their received signals as given by (2) and (3), respectively. For the full-duplex-based O-DSTC scheme, we consider that U1 and U2 will acknowledge each other and the destination if they succeed in decoding or not using feedback channels. It is assumed that both U1 and U2 always decode the acknowledgement information successfully, considering the fact that an acknowledgment consists of only one-bit information.

In the second time frame of block $k, \mathrm{U} 1$ and $\mathrm{U} 2$ encode and transmit $s_{1}$ and $s_{2}$ in an opportunistic manner depending on their decoded outcomes in the first frame. To be specific, if both U1 and U2 decode each other's information successfully, the Alamouti space-time coding [13] will be utilized, i.e., $-s_{2}^{*}$ and $s_{1}^{*}$ are transmitted by $\mathrm{U} 1$ and $\mathrm{U} 2$, respectively. Otherwise, $\mathrm{U} 1$ and $\mathrm{U} 2$, respectively, transmit $-s_{1}$ and $s_{2}$ to the destination, instead of the Alamouti coding. This is due to the fact that, when either U1 or U2 fails to decode, the use of Alamouti space-time code will introduce interference at the destination in decoding $s_{1}$ and $s_{2}$. Meanwhile, the destination can not rely on its received signal in the first frame to decode $s_{1}$ and $s_{2}$, since two unknowns $\left(s_{1}\right.$ and $\left.s_{2}\right)$ are in one equation, as shown in (1). In order to recover $s_{1}$ and $s_{2}$ at the destination in this case, U1 and U2 are allowed to transmit $-s_{1}$ and $s_{2}$, respectively, to the destination in the second time frame, which guarantees the full multiplexing gain achieved and has the advantage of simple implementation for decoding $s_{1}$ and $s_{2}$ at the destination. The coding/transmission approach discussed above is refereed to as the full-duplex-based opportunistic distributed space-time coding, which differs from the traditional DSTC in [18] and [19] where the Alamouti space-time coding is always used. With the coherent detection, the mutual information from $\mathrm{U} 2$ to $\mathrm{U} 1$ as denoted by $I_{21}$ can be calculated from (2) as

$$
I_{21}=\log _{2}\left(1+\frac{\left|h_{21}\right|^{2} \gamma}{2}\right)
$$

where $\gamma=\frac{E_{s}}{N_{0}}$. Similarly, from (3), the mutual information from $\mathrm{U} 1$ to $\mathrm{U} 2$ is given by

$$
I_{12}=\log _{2}\left(1+\frac{\left|h_{12}\right|^{2} \gamma}{2}\right) .
$$

In an information-theoretic sense, when the channel capacity falls below a predefined data rate, it is regarded as an outage event and the receiver is doomed to fail to decode the original data no matter what decoding algorithm is used. Hence, considering data rates $R_{1}$ and $R_{2}$ (for $\mathrm{U} 1$ and $\mathrm{U} 2$, respectively), the event that both $U 1$ and $U 2$ succeed in decoding can be described as $I_{12}>R_{1}$ and $I_{21}>R_{2}$, which is denoted by $\theta=1$ for notation convenience. Similarly, we use $\theta=2$ to represent the other case that either $\mathrm{U} 1$ or $\mathrm{U} 2$ or both fail to decode, i.e., $I_{12}<R_{1}$ and/or $I_{21}<R_{2}$. In the case of $\theta=1$, the Alamouti space-time coding will be utilized, and $-s_{2}^{*}$ and $s_{1}^{*}$ are transmitted by U1 and $\mathrm{U} 2$ in the second time frame of block $k$. Thus, the received signal at the destination is written as

$$
y_{d}^{2}(\theta=1)=-\sqrt{\frac{E_{s}}{2}} h_{1 d} s_{2}^{*}+\sqrt{\frac{E_{s}}{2}} h_{2 d} s_{1}^{*}+n_{d}^{2}
$$

where the superscript 2 represents the second time frame and $n_{d}^{2}$ is the AWGN received at destination. Combining (1) and (6), we can obtain from Alamouti decoding algorithm as

$$
\begin{aligned}
& {\left[\begin{array}{cc}
h_{1 d}^{*} & h_{2 d} \\
h_{2 d}^{*} & -h_{1 d}
\end{array}\right]\left[\begin{array}{c}
y_{d}^{1} \\
y_{d}^{2}(\theta=1)^{*}
\end{array}\right]} \\
& \quad=\sqrt{\frac{E_{s}}{2}}\left[\begin{array}{cc}
\left|h_{1 d}\right|^{2}+\left|h_{2 d}\right|^{2} & 0 \\
0 & \left|h_{1 d}\right|^{2}+\left|h_{2 d}\right|^{2}
\end{array}\right]\left[\begin{array}{l}
s_{1} \\
s_{2}
\end{array}\right] \\
& \quad+\left[\begin{array}{l}
h_{1 d}^{*} n_{d}^{1}+h_{2 d}\left(n_{d}^{2}\right)^{*} \\
h_{2 d}^{*} n_{d}^{1}-h_{1 d}\left(n_{d}^{2}\right)^{*}
\end{array}\right] .
\end{aligned}
$$


Hence, in the case of $\theta=1$, the mutual information from $\mathrm{U} 1$ and $\mathrm{U} 2$ to the destination is calculated from (7) as

$$
I_{1 d}(\theta=1)=I_{2 d}(\theta=1)=\log _{2}\left(1+\frac{\left|h_{1 d}\right|^{2}+\left|h_{2 d}\right|^{2}}{2} \gamma\right)
$$

Given case $\theta=2$ occurred, i.e., either U1 or U2 or both fail to decode each other's information, $\mathrm{U} 1$ and $\mathrm{U} 2$, respectively, transmit $-s_{1}$ and $s_{2}$ to the destination. Thus, in the case of $\theta=$ 2 , the received signal at the destination in the second time frame is given by

$$
y_{d}^{2}(\theta=2)=-\sqrt{\frac{E_{s}}{2}} h_{1 d} s_{1}+\sqrt{\frac{E_{s}}{2}} h_{2 d} s_{2}+n_{d}^{2}
$$

By solving (1) and (9), the destination can easily decode $s_{1}$ and $s_{2}$ as follows

$$
\begin{aligned}
{\left[\begin{array}{cc}
h_{1 d}^{*} & 0 \\
0 & h_{2 d}^{*}
\end{array}\right]\left[\begin{array}{c}
y_{d}^{1}-y_{d}^{2}(\theta=2) \\
y_{d}^{1}+y_{d}^{2}(\theta=2)
\end{array}\right]=} & {\left[\begin{array}{l}
\sqrt{2 E_{s}}\left|h_{1 d}\right|^{2} s_{1} \\
\sqrt{2 E_{s}}\left|h_{2 d}\right|^{2} s_{2}
\end{array}\right] } \\
& +\left[\begin{array}{l}
h_{1 d}^{*}\left(n_{d}^{1}-n_{d}^{2}\right) \\
h_{2 d}^{*}\left(n_{d}^{1}+n_{d}^{2}\right)
\end{array}\right]
\end{aligned}
$$

from which $s_{1}$ and $s_{2}$ are estimated as $\hat{s}_{1}=$ $\arg \min _{s \in \mathcal{S}_{1}}\left|h_{1 d}^{*}\left[y_{d}^{1}-y_{d}^{2}(\theta=2)-h_{1 d} \sqrt{2 E_{s}} s\right]\right|^{2} \quad$ and

$\hat{s}_{2}=\arg \min _{s \in \mathcal{S}_{2}}\left|h_{2 d}^{*}\left[y_{d}^{1}+y_{d}^{2}(\theta=2)-h_{2 d} \sqrt{2 E_{s}} s\right]\right|^{2}$ by using the maximum likelihood (ML) detection, where $\mathcal{S}_{1}$ and $\mathcal{S}_{2}$, respectively, represent the sets of modulation symbols $s_{1}$ and $s_{2}$. Therefore, given $\theta=2$, the mutual information from U1 and $\mathrm{U} 2$ to the destination, $I_{1 d}(\theta=2)$ and $I_{2 d}(\theta=2)$, are obtained from (10) as

$$
I_{1 d}(\theta=2)=\log _{2}\left(1+\left|h_{1 d}\right|^{2} \gamma\right)
$$

and

$$
I_{2 d}(\theta=2)=\log _{2}\left(1+\left|h_{2 d}\right|^{2} \gamma\right) .
$$

Although the occurrence of case $\theta=2$ results in both $s_{1}$ and $s_{2}$ going through only one fading path as shown in (11) and (12), the case $\theta=2$ occurs when either U1 or U2 fails to decode its partner's information, i.e., one of the two inter-user channels must be in outage given $\theta=2$. Hence, the destination fails to decode $s_{1}$ (or $s_{2}$ ) only when the channel from U1 to destination and one of the inter-user channels are both in outage. This implies that, in the case of $\theta=2$, both $\mathrm{U} 1$ and $\mathrm{U} 2$ can still achieve a diversity gain of two. In addition, it is pointed out that, by considering that both $\mathrm{U} 1$ and $\mathrm{U} 2$ notify the destination whether or not they succeed in decoding each other's information through feedback channels, the destination is able to determine which detection algorithm should be selected between (7) and (10) and used for decoding $s_{1}$ and $s_{2}$.

\section{Half-Duplex-Based O-DSTC Scheme}

This subsection discusses the half-duplex-based O-DSTC scheme. One can see from Fig. 2(b) that in the half-duplex-based O-DSTC scheme, three time frames within one block are required to transmit two symbols $s_{1}$ and $s_{2}$. In order to send the same amount information as the noncooperative scheme during one block, the half-duplex-based O-DSTC scheme shall transmit at 1.5 times data rate of the noncooperative transmission. Thus, we consider U1 and U2 with data rates $\frac{3 R_{1}}{2}$ and $\frac{3 R_{2}}{2}$ in bits per frame, respectively, for the half-duplex-based O-DSTC. In addition, as shown in Fig. 2(b), the half-duplex-based O-DSTC scheme divides one block into three time frames and requires both $\mathrm{U} 1$ and $\mathrm{U} 2$ to transmit in either one or two frames per block. Assuming the worst case of the two users transmitting in two frames per block, we consider the power of $\frac{3 E_{s}}{4}$ for each user during one time frame in the half-duplex-based O-DSTC scheme for a fair comparison with the noncooperative scheme in terms of power consumption.

In the first time frame of block $k, \mathrm{U} 1$ broadcasts its signal $s_{1}$ with power $\frac{3 E_{s}}{4}$ and rate $\frac{3 R_{1}}{2}$ to $\mathrm{U} 2$ and destination. Thus, the received signals at the destination and $\mathrm{U} 2$ are expressed as

$$
y_{d}^{1}=\sqrt{\frac{3 E_{s}}{4}} h_{1 d} s_{1}+n_{d}^{1}
$$

and

$$
y_{2}=\sqrt{\frac{3 E_{s}}{4}} h_{12} s_{1}+n_{2} .
$$

Similarly, in the second time frame, U2 transmits $s_{2}$ to U1 and destination with power $\frac{3 E_{s}}{4}$ and rate $\frac{3 R_{2}}{2}$. Hence, the received signals at the destination and $\mathrm{U} 1$ are given by

$$
y_{d}^{2}=\sqrt{\frac{3 E_{s}}{4}} h_{2 d} s_{2}+n_{d}^{2}
$$

and

$$
y_{1}=\sqrt{\frac{3 E_{s}}{4}} h_{21} s_{2}+n_{1} .
$$

Then, $\mathrm{U} 1$ and $\mathrm{U} 2$ attempt to decode their received signals based on (14) and (16), respectively. In the third time frame of block $k$, the transmit symbols sent by $\mathrm{U} 1$ and $\mathrm{U} 2$ depend on their local decoded outcome without any acknowledgment (feedback) between each other. Specifically, if U1 succeeds in decoding U2's information $s_{2}$, it will transmit $-s_{2}^{*}$; otherwise, it just keeps quiet to avoid interference. Similarly, if U2 succeeds in decoding $s_{1}$, it will transmit $s_{1}^{*}$ to the destination; otherwise, no signal is transmitted. Hence, there are four possible outcomes at the destination which requires respective decoding algorithms. As shown in Fig. 3, we can implement four decoding branches in parallel at the destination and, in general, only one branch output will pass the forward error detection (e.g., CRC checking). This means that the destination can decode $s_{1}$ and $s_{2}$ locally without any feedback information from U1 and U2 about whether the two users decode each other's information successfully or not. This advantage comes at the cost of implementation complexity due to the parallel decoding architecture. It is pointed out that, if feedback channels are available for U1 and U2 to notify the destination whether they succeed in decoding or not, the multiple parallel decoding branches as illustrated in Fig. 3 can be reduced to a single branch structure.

With the coherent detection, the mutual information from $\mathrm{U} 2$ to U1 and that from U1 to U2 are calculated from (14) and (16) as

$$
I_{12}=\log _{2}\left(1+\frac{3\left|h_{12}\right|^{2} \gamma}{4}\right)
$$




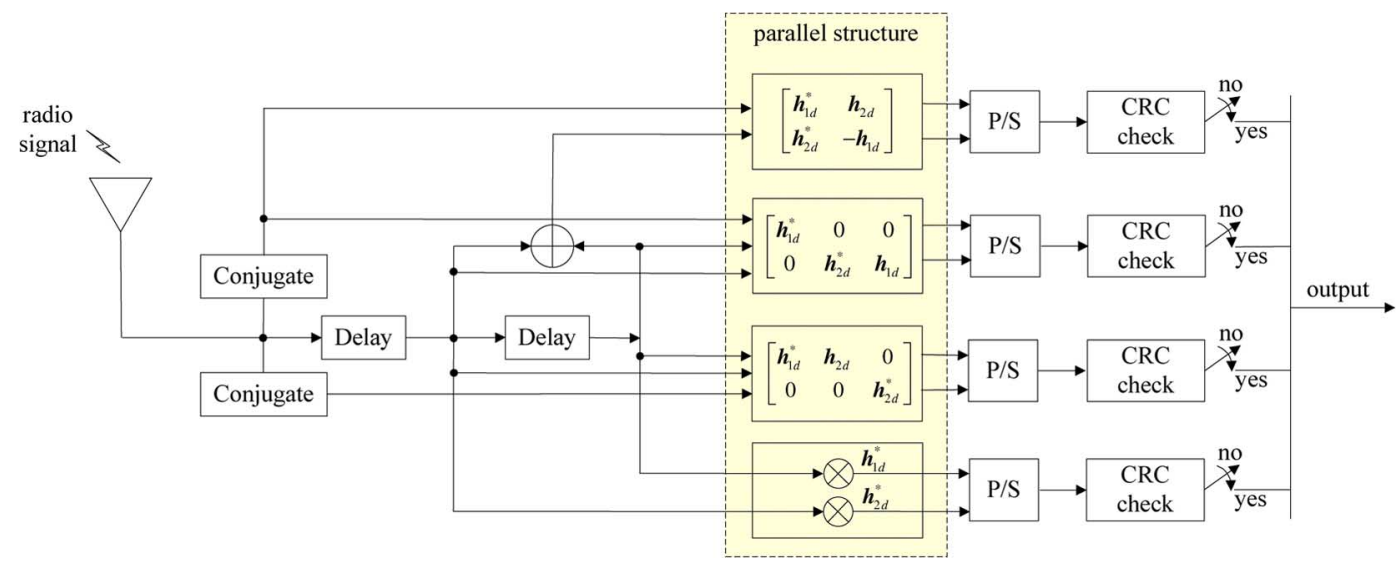

Fig. 3. A fully distributed approach for implementation of the proposed half-duplex-based O-DSTC decoding at the destination.

and

$$
I_{21}=\log _{2}\left(1+\frac{3\left|h_{21}\right|^{2} \gamma}{4}\right)
$$

As discussed above, there are four possible outcomes with regard to whether $\mathrm{U} 1$ and $\mathrm{U} 2$ succeed in decoding each other. For simplicity, let $\omega=1,2,3$, and 4, respectively, denote U1 and U2 decode successfully, U1 succeeds and U2 fails, U1 fails and $\mathrm{U} 2$ succeeds, and both fail. Hence, in an information-theoretic sense, events $\omega=1,2,3$, and 4 are described as

$$
\begin{array}{lllll}
\omega=1: & I_{12}>\frac{3 R_{1}}{2} & \text { and } & I_{21}>\frac{3 R_{2}}{2} \\
\omega=2: & I_{12}<\frac{3 R_{1}}{2} & \text { and } & I_{21}>\frac{3 R_{2}}{2} \\
\omega=3: & I_{12}>\frac{3 R_{1}}{2} & \text { and } & I_{21}<\frac{3 R_{2}}{2} \\
\omega=4: & I_{12}<\frac{3 R_{1}}{2} \quad \text { and } & I_{21}<\frac{3 R_{2}}{2} .
\end{array}
$$

In the case of $\omega=1,-s_{2}^{*}$ and $s_{1}^{*}$ are transmitted by $\mathrm{U} 1$ and $\mathrm{U} 2$ in the third time frame of block $k$. Thus, the received signal at the destination in this case is written as

$$
y_{d}^{3}(\omega=1)=-\sqrt{\frac{3 E_{s}}{4}} h_{1 d} s_{2}^{*}+\sqrt{\frac{3 E_{s}}{4}} h_{2 d} s_{1}^{*}+n_{d}^{3}
$$

where $n_{d}^{3}$ is AWGN with zero mean and variance $N_{0}$. By using (13), (15) and (20), $s_{1}$ and $s_{2}$ are demodulated at the destination as follows

$$
\begin{aligned}
& {\left[\begin{array}{cc}
h_{1 d}^{*} & h_{2 d} \\
h_{2 d}^{*} & -h_{1 d}
\end{array}\right]\left[\begin{array}{c}
y_{d}^{1}+y_{d}^{2} \\
y_{d}^{3}(\omega=1)^{*}
\end{array}\right]} \\
& =\sqrt{\frac{3 E_{s}}{4}}\left[\begin{array}{cc}
\left|h_{1 d}\right|^{2}+\left|h_{2 d}\right|^{2} & 0 \\
0 & \left|h_{1 d}\right|^{2}+\left|h_{2 d}\right|^{2}
\end{array}\right]\left[\begin{array}{l}
s_{1} \\
s_{2}
\end{array}\right] \\
& \quad+\left[\begin{array}{l}
h_{1 d}^{*} n_{d}^{\prime}+h_{2 d}\left(n_{d}^{3}\right)^{*} \\
h_{2 d}^{*} n_{d}^{\prime}-h_{1 d}\left(n_{d}^{3}\right)^{*}
\end{array}\right]
\end{aligned}
$$

where $n_{d}^{\prime}=n_{d}^{1}+n_{d}^{2}$. Note that the motivation of using $y_{d}^{1}+y_{d}^{2}$ jointly with $y_{d}^{3}(\omega=1)$ is to employ the Alamouti decoding algorithm to decode the desired signals $s_{1}$ and $s_{2}$. One can see that the first matrix in (21) is the exact Alamouti decoding matrix which is also used in (7) for the full-duplex-based O-DSTC scheme. It is pointed out that the decoding strategy adopted in (21) has low computational complexity and preserves the full diversity, as will be shown later in (66). Hence, given $\omega=1$, the mutual information from $\mathrm{U} 1$ and $\mathrm{U} 2$ to the destination, $I_{1 d}(\omega=$ $1)$ and $I_{2 d}(\omega=1)$, are calculated from (21) as

$$
\begin{aligned}
I_{1 d}(\omega=1) & =\log _{2}\left[1+\frac{3\left(\left|h_{1 d}\right|^{2}+\left|h_{2 d}\right|^{2}\right)^{2}}{4\left(2\left|h_{1 d}\right|^{2}+\left|h_{2 d}\right|^{2}\right)} \gamma\right] \\
& \geq \log _{2}\left(1+\frac{3\left(\left|h_{1 d}\right|^{2}+\left|h_{2 d}\right|^{2}\right)}{8} \gamma\right)
\end{aligned}
$$

and

$$
\begin{aligned}
I_{2 d}(\omega=1) & =\log _{2}\left[1+\frac{3\left(\left|h_{1 d}\right|^{2}+\left|h_{2 d}\right|^{2}\right)^{2}}{4\left(\left|h_{1 d}\right|^{2}+2\left|h_{2 d}\right|^{2}\right)} \gamma\right] \\
& \geq \log _{2}\left(1+\frac{3\left(\left|h_{1 d}\right|^{2}+\left|h_{2 d}\right|^{2}\right)}{8} \gamma\right) .
\end{aligned}
$$

Given $\omega=2$ occurred, i.e., U1 succeeds in decoding $s_{2}$ from (16) and $\mathrm{U} 2$ fails to decode $s_{1}$ from (14), U1 transmits $-s_{2}^{*}$ and U2 keeps quiet during the third time frame of block $k$. Therefore, in the case of $\omega=2$, the received signal at the destination is given by

$$
y_{d}^{3}(\omega=2)=-\sqrt{\frac{3 E_{s}}{4}} h_{1 d} s_{2}^{*}+n_{d}^{3} .
$$

Using (13), (15) and (24), the destination will decode $s_{1}$ and $s_{2}$ as follows

$$
\begin{aligned}
& {\left[\begin{array}{ccc}
h_{1 d}^{*} & 0 & 0 \\
0 & h_{2 d}^{*} & h_{1 d}
\end{array}\right]\left[\begin{array}{c}
y_{d}^{1} \\
y_{d}^{2} \\
y_{d}^{3}(\omega=2)^{*}
\end{array}\right]} \\
& =\sqrt{\frac{3 E_{s}}{4}}\left[\begin{array}{c}
\left|h_{1 d}\right|^{2} s_{1} \\
\left(\left|h_{1 d}\right|^{2}+\left|h_{2 d}\right|^{2}\right) s_{2}
\end{array}\right]+\left[\begin{array}{c}
h_{1 d}^{*} n_{d}^{1} \\
h_{2 d}^{*} n_{d}^{2}+h_{1 d}\left(n_{d}^{3}\right)^{*}
\end{array}\right] .
\end{aligned}
$$

Hence, given $\omega=2$, the mutual information from U1 to the destination and that from $\mathrm{U} 2$ to the destination are given by

$$
I_{1 d}(\omega=2)=\log _{2}\left(1+\frac{3\left|h_{1 d}\right|^{2} \gamma}{4}\right)
$$


and

$$
I_{2 d}(\omega=2)=\log _{2}\left[1+\frac{3\left(\left|h_{1 d}\right|^{2}+\left|h_{2 d}\right|^{2}\right)}{8} \gamma\right]
$$

Note that case $\omega=2$ occurs when the channel from U1 to U2 is in outage. Thus, the destination will fail to decode $s_{1}$ only when both the channels from $\mathrm{U} 1$ to $\mathrm{U} 2$ and that from U1 to destination are in outage. This implies that a diversity gain of two is still achieved by the U1's transmissions given $\omega=2$, which will be proven in Section IV. In the case of $\omega=3$, i.e., U1 fails to decode $s_{2}$ from (16) and U2 succeeds in decoding $s_{1}$ from (14), U1 keeps quiet and U2 transmits $s_{1}^{*}$ in the third time frame of block $k$. Hence, given $\omega=3$, the received signal at the destination can be given by

$$
y_{d}^{3}(\omega=3)=\sqrt{\frac{3 E_{s}}{4}} h_{2 d} s_{1}^{*}+n_{d}^{3} .
$$

Combining (13), (15), and (28), the destination can decode $s_{1}$ and $s_{2}$ as given by

$$
\begin{aligned}
& {\left[\begin{array}{ccc}
h_{1 d}^{*} & h_{2 d} & 0 \\
0 & 0 & h_{2 d}^{*}
\end{array}\right]\left[\begin{array}{c}
y_{d}^{1} \\
y_{d}^{3}(\omega=2)^{*} \\
y_{d}^{2}
\end{array}\right]} \\
& =\sqrt{\frac{3 E_{s}}{4}}\left[\begin{array}{c}
\left(\left|h_{1 d}\right|^{2}+\left|h_{2 d}\right|^{2}\right) s_{1} \\
\left|h_{2 d}\right|^{2} s_{2}
\end{array}\right]+\left[\begin{array}{c}
h_{1 d}^{*} n_{d}^{1}+h_{2 d}\left(n_{d}^{3}\right)^{*} \\
h_{2 d}^{*} n_{d}^{2}
\end{array}\right]
\end{aligned}
$$

from which the mutual information from U1 and U2 to the destination can be calculated as

$$
I_{1 d}(\omega=3)=\log _{2}\left[1+\frac{3\left(\left|h_{1 d}\right|^{2}+\left|h_{2 d}\right|^{2}\right)}{8} \gamma\right]
$$

and

$$
I_{2 d}(\omega=3)=\log _{2}\left(1+\frac{3\left|h_{2 d}\right|^{2} \gamma}{4}\right) .
$$

The last case $\omega=4$ indicates that both $U 1$ and $U 2$ fail to decode each other's information. In this case, the destination can only rely on (13) and (15) to decode $s_{1}$ and $s_{2}$, respectively. Thus, the corresponding mutual information from U1 and U2 to destination, $I_{1 d}(\omega=4)$ and $I_{2 d}(\omega=4)$, are given by

$$
I_{1 d}(\omega=4)=\log _{2}\left(1+\frac{3\left|h_{1 d}\right|^{2} \gamma}{4}\right)
$$

and

$$
I_{2 d}(\omega=4)=\log _{2}\left(1+\frac{3\left|h_{2 d}\right|^{2} \gamma}{4}\right) .
$$

It is worth mentioning that case $\omega=4$ occurs only when both the channels from $\mathrm{U} 1$ to $\mathrm{U} 2$ and from $\mathrm{U} 2$ to $\mathrm{U} 1$ are in outage.

\section{Conventional S-DF Cooperation}

For the comparison purpose, we now present the conventional S-DF cooperation [4]. As shown in Fig. 2(c), four time frames are required during one block to complete the transmissions of $s_{1}$ and $s_{2}$. Hence, for a fair comparison to the noncooperative scheme in terms of the data rate within one block, we consider $\mathrm{U} 1$ and $\mathrm{U} 2$ with data rates $2 R_{1}$ and $2 R_{2}$ in bits per frame, respectively. Besides, Fig. 2(c) shows that the S-DF cooperation scheme divides one block into four time frames, where both U1 and U2 transmit in two frames per block and keep silent during half of one block. Therefore, in order to make a fair comparison with the noncooperative scheme in terms of power consumption, we consider the power of $E_{s}$ for each user during one time frame in the S-DF cooperation scheme.

In the first time frame of block $k, \mathrm{U} 1$ broadcasts its signal $s_{1}$ to $\mathrm{U} 2$ and destination with power $E_{s}$ and rate $2 R_{1}$. Hence, the corresponding mutual information from $\mathrm{U} 1$ to $\mathrm{U} 2$ can be given by

$$
I_{12}=\log _{2}\left(1+\left|h_{12}\right|^{2} \gamma\right)
$$

In the second frame of block $k$, the transmit symbol sent by $\mathrm{U} 2$ depends on whether $\mathrm{U} 2$ decodes its received signal from U1 in the first frame successfully or not. To be specific, if U2 succeeds in decoding its received signal, it transmits $s_{1}$; Otherwise, nothing is transmitted. For notational convenience, let $\varpi=1$ represent that $\mathrm{U} 2$ succeeds in decoding $s_{1}$ and $\varpi=2$ represent the other case. Thus, we can easily describe events $\varpi=1$ and $\varpi=2$ as $I_{12}>2 R_{1}$ and $I_{12}<2 R_{1}$, respectively. Given $\varpi=1$ (implying that U2 decodes $s_{1}$ successfully), U2 would forward $s_{1}$ in the second time frame and thus the destination obtains two received copies of $s_{1}$. By considering maximum ratio combining (MRC), the conditional mutual information from U1 to destination in the case of $\varpi=1$ can be given by

$$
I_{1 d}(\varpi=1)=\log _{2}\left[1+\left(\left|h_{1 d}\right|^{2}+\left|h_{2 d}\right|^{2}\right) \gamma\right] .
$$

Besides, given $\varpi=2$, the destination can only rely on the transmission from U1 in the first frame to decode $s_{1}$, and thus the conditional mutual information from U1 to the destination in this case is given by

$$
I_{1 d}(\varpi=2)=\log _{2}\left(1+\left|h_{1 d}\right|^{2} \gamma\right) .
$$

Notice that similar mutual information can be obtained for the transmission of $s_{2}$ during the third and fourth time frames of block $k$, as shown in Fig. 2(c).

\section{Outage Probability Analysis Over Rayleigh FADING CHANNELS}

In this section, we examine the outage probability for both the full-duplex and half-duplex-based O-DSTC schemes as well as the conventional S-DF [4] and F-DSTC [18]. We only focus on the performance analysis of the transmission of $s_{1}$ from U1 to destination throughout this paper, and similar performance results can be obtained for the transmission of $s_{2}$ from U2 to destination. Let us first consider the traditional noncooperative scenario in a Rayleigh fading environment, where the outage probability of $\mathrm{U} 1$ 's transmissions with power $E_{s}$ and rate $R_{1}$ is given by

Pout $_{\text {non }}=\operatorname{Pr}\left\{\log _{2}\left(1+\left|h_{1 d}\right|^{2} \gamma\right)<R_{1}\right\}=1-\exp \left(-\frac{2^{R_{1}}-1}{\sigma_{1 d}^{2} \gamma}\right)$ 
where $\sigma_{1 d}^{2}=E\left(\left|h_{1 d}\right|^{2}\right)$.

\section{A. Full-Duplex Based DSTC Schemes}

1) Outage Analysis of Full-Duplex Based O-DSTC Scheme: As discussed in Section II-B, the full-duplex-based O-DSTC scheme utilizes an opportunistic encoding approach depending on whether U1 and U2 succeed in decoding each other's information. Following (8) and (11), an outage probability of the U1's transmission can be expressed as

$$
\begin{aligned}
& \text { Pout }_{\text {full-oDsTC }} \\
& =\operatorname{Pr}(\theta=1) \operatorname{Pr}(\text { outage } \mid \theta=1)+\operatorname{Pr}(\theta=2) \operatorname{Pr}(\text { outage } \mid \theta=2) \\
& =\operatorname{Pr}\left(I_{12}>R_{1}, I_{21}>R_{2}\right) \operatorname{Pr}\left[I_{1 d}(\theta=1)<R_{1}\right] \\
& \quad+\left[1-\operatorname{Pr}\left(I_{12}>R_{1}, I_{21}>R_{2}\right)\right] \operatorname{Pr}\left[I_{1 d}(\theta=2)<R_{1}\right]
\end{aligned}
$$

where $I_{21}, I_{12}, I_{1 d}(\theta=1)$, and $I_{1 d}(\theta=2)$ are, respectively, given by (4), (5), (8), and (11). The following presents closedform solutions to terms $\operatorname{Pr}\left(I_{12}>R_{1}, I_{21}>R_{2}\right), \operatorname{Pr}\left[I_{1 d}(\theta=\right.$ $\left.1)<R_{1}\right]$ and $\operatorname{Pr}\left[I_{1 d}(\theta=2)<R_{1}\right]$, respectively. Notice that random variables $\left|h_{21}\right|^{2},\left|h_{12}\right|^{2},\left|h_{1 d}\right|^{2}$, and $\left|h_{2 d}\right|^{2}$ follow exponential distributions with means $\sigma_{21}^{2}, \sigma_{12}^{2}, \sigma_{1 d}^{2}$, and $\sigma_{2 d}^{2}$, respectively, which are independent of each other. Hence, substituting (4) and (5) into term $\operatorname{Pr}\left(I_{12}>R_{1}, I_{21}>R_{2}\right)$, we easily obtain

$\operatorname{Pr}\left(I_{12}>R_{1}, I_{21}>R_{2}\right)=\exp \left[-\frac{2\left(2^{R_{1}}-1\right)}{\gamma \sigma_{12}^{2}}\right] \exp \left[-\frac{2\left(2^{R_{2}}-1\right)}{\gamma \sigma_{21}^{2}}\right]$.

Using (8), we can calculate $\operatorname{Pr}\left[I_{1 d}(\theta=1)<R_{1}\right]$ as

$$
\begin{aligned}
\operatorname{Pr} & {\left[I_{1 d}(\theta=1)<R_{1}\right] } \\
= & \operatorname{Pr}\left[\left|h_{1 d}\right|^{2}+\left|h_{2 d}\right|^{2}<\frac{2\left(2^{R_{1}}-1\right)}{\gamma}\right] \\
= & \left\{\begin{array}{cc}
1-\left[1+\frac{2\left(2^{R_{1}}-1\right)}{\sigma_{1 d}^{2} \gamma}\right] \\
\quad \times \exp \left[-\frac{2\left(2^{R_{1}}-1\right)}{\sigma_{1 d}^{2} \gamma}\right], & \sigma_{1 d}^{2}=\sigma_{2 d}^{2} \\
1-\frac{\sigma_{1 d}^{2}}{\sigma_{1 d}^{2}-\sigma_{2 d}^{2}} \exp \left[-\frac{2\left(2^{R_{1}}-1\right)}{\sigma_{1 d}^{2} \gamma}\right] & \\
-\frac{\sigma_{2 d}^{2}}{\sigma_{2 d}^{2}-\sigma_{1 d}^{2}} \exp \left[-\frac{2\left(2^{R_{1}}-1\right)}{\sigma_{2 d}^{2} \gamma}\right], & \text { otherwise. }
\end{array}\right.
\end{aligned}
$$

Similarly, substituting (11) into term $\operatorname{Pr}\left[I_{1 d}(\theta=2)<R_{1}\right]$, we easily obtain

$$
\operatorname{Pr}\left[I_{1 d}(\theta=2)<R_{1}\right]=\left[1-\exp \left(-\frac{2^{R_{1}}-1}{\sigma_{`}^{2} 1 d}\right)\right] .
$$

Now, we complete the closed-form outage probability analysis for the full-duplex-based O-DSTC scheme. In what follows, we examine the outage performance of the traditional F-DSTC [18] with full-duplex regime, called the full-duplex-based F-DSTC.

2) Outage Analysis of Full-Duplex Based F-DSTC Scheme: Typically, the F-DSTC scheme proposed in [18] always adopts the so-called distributed Alamouti space-time coding to achieve the cooperative diversity, no matter whether U1 and U2 decode each other's information successfully or not. To be specific, in the first time frame of block $k$, both $\mathrm{U} 1$ and $\mathrm{U} 2$ transmit their own information $s_{1}$ and $s_{2}$ to the destination with power $\frac{E_{s}}{2}$. Considering the full-duplex regime, $\mathrm{U} 1$ and $\mathrm{U} 2$ can receive and decode each other's information, where the decoded outcomes at $\mathrm{U} 1$ and $\mathrm{U} 2$ are denoted by $\hat{s}_{2}$ and $\hat{s}_{1}$, respectively. Then, in the subsequent time frame, U1 and U2 transmit their decoded outcomes according to the Alamouti space-time coding, i.e., $-\hat{s}_{2}^{*}$ and $\hat{s}_{1}^{*}$ are forwarded to the destination. Thus, the signal vector received at the destination in two consecutive time frames of block $k$, as denoted by $\left[y_{d}^{1} y_{d}^{2}\right]^{T}$, can be written as

$$
\left[\begin{array}{l}
y_{d}^{1} \\
y_{d}^{2}
\end{array}\right]=\sqrt{\frac{E_{s}}{2}}\left[\begin{array}{c}
h_{1 d} s_{1}+h_{2 d} s_{2} \\
-h_{1 d} \hat{s}_{2}^{*}+h_{2 d} \hat{s}_{1}^{*}
\end{array}\right]+\left[\begin{array}{l}
n_{d}^{1} \\
n_{d}^{2}
\end{array}\right]
$$

which can be further rewritten as

$$
\begin{aligned}
{\left[\begin{array}{c}
y_{d}^{1} \\
\left(y_{d}^{2}\right)^{*}
\end{array}\right]=} & \sqrt{\frac{E_{s}}{2}}\left[\begin{array}{c}
h_{1 d} s_{1}+h_{2 d} s_{2} \\
-h_{1 d}^{*}\left(s_{2}+\hat{s}_{2}-s_{2}\right)+h_{2 d}^{*}\left(s_{1}+\hat{s}_{1}-s_{1}\right)
\end{array}\right] \\
& +\left[\begin{array}{c}
n_{d}^{1} \\
\left(n_{d}^{2}\right)^{*}
\end{array}\right] \\
= & \sqrt{\frac{E_{s}}{2}}\left[\begin{array}{cc}
h_{1 d} & h_{2 d} \\
h_{2 d}^{*} & -h_{1 d}^{*}
\end{array}\right]\left[\begin{array}{c}
s_{1} \\
s_{2}
\end{array}\right] \\
& +\left[\begin{array}{cc}
n_{d}^{*} \\
h_{2 d}^{*}\left(\hat{s}_{1}-s_{1}\right)-h_{1 d}^{*}\left(\hat{s}_{2}-s_{2}\right)+\left(n_{d}^{2}\right)^{*}
\end{array}\right] \cdot
\end{aligned}
$$

By applying the Alamouti decoding to (43), the destination attempts to decode $s_{1}$ and $s_{2}$ as shown in (44) at the bottom of the page. One can observe from the second term in the right-hand side (RHS) of (44) that either U1 or U2 or both failing to decode will lead to $\hat{s}_{1} \neq s_{1}$ and/or $\hat{s}_{2} \neq s_{2}$, which results in interference at the destination in decoding both $s_{1}$ and $s_{2}$, and severely degrades the transmission performance. This also implies that the interuser channels between $\mathrm{U} 1$ and $\mathrm{U} 2$ are the bottleneck of the traditional F-DSTC scheme. It will be shown in Section IV that the F-DSTC scheme can not achieve the full diversity due to the bottleneck effect caused by inter-user channels.

From (44), when both U1 and U2 succeed in decoding, i.e., $I_{12}>R_{1}$ and $I_{21}>R_{2}$, we have $\hat{s}_{1}=s_{1}$ and $\hat{s}_{2}=s_{2}$, thus the mutual information from U1 to the destination is given by $\log _{2}\left(1+\frac{\left(\left|h_{1 d^{\prime 2}}+\right| h_{2 d^{\prime 2}}\right) \gamma}{2}\right)$. When U1 succeeds and U2 fails, i.e., $I_{12}<R_{1}$ and

$$
\begin{array}{r}
{\left[\begin{array}{cc}
h_{1 d}^{*} & h_{2 d} \\
h_{2 d}^{*} & -h_{1 d}
\end{array}\right]\left[\begin{array}{c}
y_{d}^{1} \\
\left(y_{d}^{2}\right)^{*}
\end{array}\right]=\sqrt{\frac{E_{s}}{2}}\left[\begin{array}{cc}
\left|h_{1 d}\right|^{2}+\left|h_{2 d}\right|^{2} & 0 \\
0 & \left|h_{1 d}\right|^{2}+\left|h_{2 d}\right|^{2}
\end{array}\right]\left[\begin{array}{c}
s_{1} \\
s_{2}
\end{array}\right]} \\
+\left[\begin{array}{c}
\left|h_{2 d}\right|^{2}\left(\hat{s}_{1}-s_{1}\right)-h_{1 d}^{*} h_{2 d}\left(\hat{s}_{2}-s_{2}\right)+h_{1 d}^{*} n_{d}^{1}+h_{2 d}\left(n_{d}^{2}\right)^{*} \\
-h_{1 d} h_{2 d}^{*}\left(\hat{s}_{1}-s_{1}\right)+\left|h_{2 d}\right|^{2}\left(\hat{s}_{2}-s_{2}\right)+h_{2 d}^{*} n_{d}^{1}-h_{1 d}\left(n_{d}^{2}\right)^{*}
\end{array}\right] .
\end{array}
$$


$I_{21}>R_{2}$, we have $\hat{s}_{1} \neq s_{1}$ and $\hat{s}_{2}=s_{2}$, using which the mutual information from U1 to the destination is obtained as $\log _{2}\left(1+\frac{\left(\left|h_{1 d}\right|^{2}+\left|h_{2 d}\right|^{2}\right)^{2} \gamma}{2\left(\left|h_{2 d}\right|^{4} \gamma+\left|h_{1 d}\right|^{2}+\left|h_{2 d}\right|^{2}\right)}\right)$ by considering $E\left(\left|\hat{s}_{1}-s_{1}\right|^{2}\right)=E\left(\left|\hat{s}_{1}\right|^{2}\right)+E\left(\left|s_{1}\right|^{2}\right)-E\left(\hat{s}_{1} s_{1}^{*}\right)-E\left(\hat{s}_{1}^{*} s_{1}\right)=E_{s}$ given $I_{12}<R_{1}$, where $E\left(\hat{s}_{1} s_{1}^{*}\right)=E\left(\hat{s}_{1}\right) E\left(s_{1}^{*}\right)=0$ and $E\left(\hat{s}_{1}^{*} s_{1}\right)=E\left(\hat{s}_{1}^{*}\right) E\left(s_{1}\right)=0$ arise from the fact that $\hat{s}_{1}$ and $s_{1}$ are independent of each other in case of $I_{12}<R_{1}$. Similarly, if U1 fails and $\mathrm{U} 2$ succeeds (implying $I_{12}>R_{1}$ and $I_{21}<R_{2}$ ), we obtain $\hat{s}_{1}=s_{1}$ and $\hat{s}_{2} \neq s_{2}$, thus the mutual information from $\mathrm{U} 1$ to the destination can be obtained as $\log _{2}\left(1+\frac{\left(\left|h_{1 d}\right|^{2}+\mid h_{2 d}{ }^{\prime 2}\right)^{2} \gamma}{2\left(\left|h_{1 d}\right|^{2}\left|h_{2 d}\right|^{2} \gamma+\left|h_{1 d}\right|^{2}+\left|h_{2 d}\right|^{2}\right)}\right)$. In addition, when both U1 and U2 fail to decode each other's information, i.e., $I_{12}<R_{1}$ and $I_{21}<R_{2}$, we can calculate the mutual information from U1 to the destination from (44) as $\log _{2}\left(1+\frac{\left(\left|h_{1 d}\right|^{2}+\left|h_{2 d}\right|^{2}\right)^{2} \gamma}{2\left(h_{2 d}{ }^{4} \gamma+\left|h_{1 d}\right|^{2}\left|h_{2 d}\right|^{2} \gamma+\left.h_{1 d}\right|^{2}+\left|h_{2 d}\right|^{2}\right)}\right)$. Thus, an outage probability of the full-duplex-based F-DSTC scheme is given by (45) at the bottom of the page, where $I_{21}$ and $I_{12}$ are given by (4) and (5), respectively. One can see from (45) that it is challenging to obtain a closed-form solution to the outage probability of the full-duplex-based F-DSTC scheme. Nevertheless, given a parameter set $\left\{R_{1}, R_{2}, \sigma_{12}^{2}, \sigma_{21}^{2}, \sigma_{1 d}^{2}, \sigma_{2 d}^{2}\right\}$, the outage probability can be easily calculated from (45) through numerical computation.

3) Numerical Results: We present the outage probability comparison of the proposed full-duplex-based O-DSTC scheme with the traditional noncooperative and the full-duplex-based F-DSTC schemes. In Fig. 4, we show the outage probability performance versus transmit SNR $\gamma$ of the noncooperative, the full-duplex-based F-DSTC and O-DSTC schemes with $R_{1}=R_{2}=1 \mathrm{bit} / \mathrm{s} / \mathrm{H} \%$. As shown in Fig. 4 , the proposed O-DSTC scheme strictly outperforms both the noncooperative and the F-DSTC schemes in terms of the outage probability across the whole SNR region. One can also see from Fig. 4 that, in the low SNR region, the outage probability of the F-DSTC scheme is even worse than that of the noncooperative transmission. On the other hand, in high SNR region, as the transmit SNR increases, the outage probability of the F-DSTC scheme decreases at the same speed as the noncooperative transmission. However, the outage probability decrease of the proposed O-DSTC scheme is at higher speed than both the

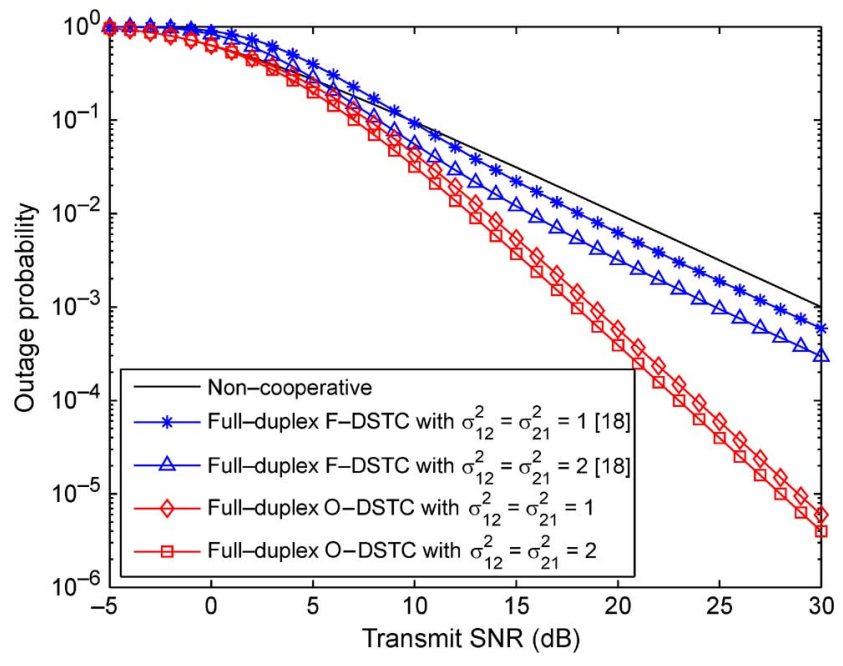

Fig. 4. Outage probability versus transmit $\operatorname{SNR}(\gamma)$ of the noncooperative, the full-duplex-based F-DSTC and O-DSTC schemes with $R_{1}=R_{2}=1 \mathrm{bit} / \mathrm{s} / \mathrm{Hz}$ and $\sigma_{1 d}^{2}=\sigma_{2 d}^{2}=1$.

F-DSTC and noncooperative schemes. This implies that the proposed O-DSTC achieves higher diversity order (also known as diversity gain), which will be proven in Section IV.

Now, we study an impact of data rates, i.e., $R_{1}$ and $R_{2}$, on outage performance through numerical evaluation by introducing multiplexing gains $r_{1}$ and $r_{2}$ (for $\mathrm{U} 1$ and $\mathrm{U} 2$, respectively), where the multiplexing gains are defined as $r_{1}=\frac{R_{1}}{\log _{2}(1+\gamma)}$ and $r_{2}=\frac{R_{2}}{\log _{2}(1+\gamma)}$. Fig. 5 shows the outage probability versus transmit SNR $\gamma$ of the noncooperative and proposed full-duplex-based O-DSTC schemes with $R_{1}=r_{1} \log _{2}(1+\gamma)$ and $R_{2}=r_{2} \log _{2}(1+\gamma)$, differing from Fig. 4 where the data rates $R_{1}$ and $R_{2}$ are set to be fixed and do not vary with the change of SNR $\gamma$. From Fig. 5, one can observe that, in the case of $r_{2}=1$, the outage probability of the O-DSTC scheme decreases at the same speed as the noncooperative in high SNR region, which shows that no diversity gain is achieved by U1's transmissions given the U2's multiplexing gain $r_{2}=1$. As $r_{2}$ decreases from $r_{2}=1$ to 0.4 , the speed of the outage probability decrease in high SNR region improves and, moreover, it keeps unchanged when $r_{2}$ decreases from

$$
\begin{aligned}
\text { Pout }_{\text {full-FDSTC }}= & \operatorname{Pr}\left(I_{12}>R_{1}, I_{21}>R_{2}\right) \operatorname{Pr}\left[\log _{2}\left(1+\frac{\left(\left|h_{1 d}\right|^{2}+\left|h_{2 d}\right|^{2}\right) \gamma}{2}\right)<R_{1}\right] \\
& +\operatorname{Pr}\left(I_{12}<R_{1}, I_{21}>R_{2}\right) \operatorname{Pr}\left[\log _{2}\left(1+\frac{\left(\left|h_{1 d}\right|^{2}+\left|h_{2 d}\right|^{2}\right)^{2} \gamma}{2\left|h_{2 d}\right|^{4} \gamma+2\left(\left|h_{1 d}\right|^{2}+\left|h_{2 d}\right|^{2}\right)}\right)<R_{1}\right] \\
& +\operatorname{Pr}\left(I_{12}>R_{1}, I_{21}<R_{2}\right) \operatorname{Pr}\left[\log _{2}\left(1+\frac{\left(\left|h_{1 d}\right|^{2}+\left|h_{2 d}\right|^{2}\right)^{2} \gamma}{2\left|h_{1 d}\right|^{2}\left|h_{2 d}\right|^{2} \gamma+2\left(\left|h_{1 d}\right|^{2}+\left|h_{2 d}\right|^{2}\right)}\right)<R_{1}\right] \\
& +\operatorname{Pr}\left(I_{12}<R_{1}, I_{21}<R_{2}\right) \operatorname{Pr}\left[\log _{2}\left(1+\frac{\left(\left|h_{1 d}\right|^{2}+\left|h_{2 d}\right|^{2}\right)^{2} \gamma}{2\left(\left|h_{2 d}\right|^{4}+\left|h_{1 d}\right|^{2}\left|h_{2 d}\right|^{2}\right) \gamma+2\left(\left|h_{1 d}\right|^{2}+\left|h_{2 d}\right|^{2}\right)}\right)<R_{1}\right]
\end{aligned}
$$




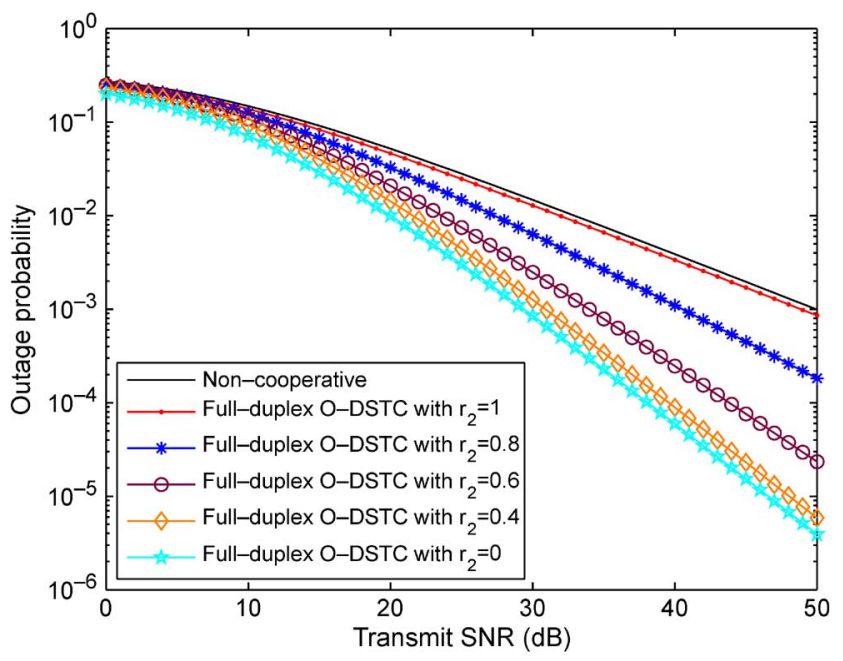

Fig. 5. Outage probability versus transmit $\operatorname{SNR}(\gamma)$ of the noncooperative and proposed full-duplex-based O-DSTC schemes for different U2's multiplexing gains $\left(r_{2}\right)$ with U1's multiplexing gain $r_{1}=0.4$ and $\sigma_{12}^{2}=\sigma_{1 d}^{2}=\sigma_{21}^{2}=$ $\sigma_{2 d}^{2}=1$.

$r_{2}=0.4$ to 0 . In other words, given U1's multiplexing gain $r_{1}=0.4$, the diversity gain obtained by U1's transmissions initially increases, as $r_{2}$ decreases from $r_{2}=1$ to 0.4 , and eventually converges after $r_{2}<0.4$. Therefore, one can conclude that the diversity gain of U1 in the full-duplex-based O-DSTC scheme relates to the multiplexing gains of both $\mathrm{U} 1$ and $\mathrm{U} 2$.

\section{B. Half-Duplex Based DSTC Schemes}

1) Outage Analysis of Half-Duplex Based O-DSTC: In this subsection, we study the outage probability performance of the proposed half-duplex-based O-DSTC scheme. Following (19), (22), (26), (30), and (32), an outage probability of the U1's transmission with the half-duplex-based O-DSTC scheme is calculated as

$$
\begin{aligned}
\text { Pout }_{\text {half-ODSTC }}= & \operatorname{Pr}(\omega=1) \operatorname{Pr}\left[I_{1 d}(\omega=1)<\frac{3 R_{1}}{2}\right] \\
& +\operatorname{Pr}(\omega=2) \operatorname{Pr}\left[I_{1 d}(\omega=2)<\frac{3 R_{1}}{2}\right] \\
& +\operatorname{Pr}(\omega=3) \operatorname{Pr}\left[I_{1 d}(\omega=3)<\frac{3 R_{1}}{2}\right] \\
& +\operatorname{Pr}(\omega=4) \operatorname{Pr}\left[I_{1 d}(\omega=4)<\frac{3 R_{1}}{2}\right] .
\end{aligned}
$$

In the following, we examine closed-form solutions to these terms as given in the RHS of the above equation. Combining (17)-(19), we can easily obtain closed-form solutions to terms $\operatorname{Pr}(\omega=1), \operatorname{Pr}(\omega=2), \operatorname{Pr}(\omega=3)$, and $\operatorname{Pr}(\omega=4)$ as

$$
\begin{aligned}
\operatorname{Pr}(\omega=1)= & \exp \left[-\frac{4\left(2^{3 R_{1} / 2}-1\right)}{3 \gamma \sigma_{12}^{2}}\right] \exp \left[-\frac{4\left(2^{3 R_{2} / 2}-1\right)}{3 \gamma \sigma_{21}^{2}}\right] \\
\operatorname{Pr}(\omega=2)= & {\left[1-\exp \left(-\frac{4\left(2^{3 R_{1} / 2}-1\right)}{3 \gamma \sigma_{12}^{2}}\right)\right] } \\
& \times \exp \left[-\frac{4\left(2^{3 R_{2} / 2}-1\right)}{3 \gamma \sigma_{21}^{2}}\right] \\
\operatorname{Pr}(\omega=3)= & \exp \left[-\frac{4\left(2^{3 R_{1} / 2}-1\right)}{3 \gamma \sigma_{12}^{2}}\right]
\end{aligned}
$$

$$
\begin{aligned}
& \times\left[1-\exp \left(-\frac{4\left(2^{3 R_{2} / 2}-1\right)}{3 \gamma \sigma_{21}^{2}}\right)\right] \\
\operatorname{Pr}(\omega=4)= & {\left[1-\exp \left(-\frac{4\left(2^{3 R_{1} / 2}-1\right)}{3 \gamma \sigma_{12}^{2}}\right)\right] } \\
& \times\left[1-\exp \left(-\frac{4\left(2^{3 R_{2} / 2}-1\right)}{3 \gamma \sigma_{21}^{2}}\right)\right] .
\end{aligned}
$$

By using (22) and (30), a closed-form expression for $\operatorname{Pr}\left[I_{1 d}(\omega=1)<\frac{3 R_{1}}{2}\right]$ and $\operatorname{Pr}\left[I_{1 d}(\omega=3)<\frac{3 R_{1}}{2}\right]$ is given by

$$
\begin{aligned}
& \operatorname{Pr}\left[I_{1 d}(\omega=1)<\frac{3 R_{1}}{2}\right] \\
& =\operatorname{Pr}\left[I_{1 d}(\omega=3)<\frac{3 R_{1}}{2}\right] \\
& =\left\{\begin{array}{cc}
1-\left[1+\frac{8\left(2^{3 R_{1} / 2}-1\right)}{3 \sigma_{1 d}^{2} \gamma}\right] \\
\quad \times \exp \left(-\frac{8\left(2^{3 R_{1} / 2}-1\right)}{3 \sigma_{1 d}^{2} \gamma}\right), & \sigma_{1 d}^{2}=\sigma_{2 d}^{2} \\
1-\frac{\sigma_{1 d}^{2}}{\sigma_{1 d}^{2}-\sigma_{2 d}^{2}} \exp \left(-\frac{8\left(2^{3 R_{1} / 2}-1\right)}{3 \sigma_{1 d}^{2} \gamma}\right) & \\
-\frac{\sigma_{2 d}^{2}}{\sigma_{2 d}^{2}-\sigma_{1 d}^{2}} \exp \left(-\frac{8\left(2^{3 R_{1} / 2}-1\right)}{3 \sigma_{2 d}^{2} \gamma}\right), & \text { otherwise. }
\end{array}\right.
\end{aligned}
$$

Similarly, following (26) and (32), we can calculate terms $\operatorname{Pr}\left[I_{1 d}(\omega=2)<\frac{3 R_{1}}{2}\right]$ and $\operatorname{Pr}\left[I_{1 d}(\omega=4)<\frac{3 R_{1}}{2}\right]$ as

$$
\begin{aligned}
\operatorname{Pr}\left[I_{1 d}(\omega=2)<\frac{3 R_{1}}{2}\right] & =\operatorname{Pr}\left[I_{1 d}(\omega=4)<\frac{3 R_{1}}{2}\right] \\
& =1-\exp \left[-\frac{4\left(2^{3 R_{1} / 2}-1\right)}{3 \gamma \sigma_{1 d}^{2}}\right] .
\end{aligned}
$$

This completes a closed-form outage probability analysis for the proposed half-duplex-based O-DSTC scheme. In what follows, we present an outage analysis of the traditional F-DSTC scheme with the half-duplex relaying, referred to as the half-duplexbased F-DSTC.

2) Outage Analysis of Half-Duplex Based F-DSTC Scheme: The difference between the half-duplex-based F-DSTC and the proposed half-duplex-based O-DSTC lies in the third time frame of block $k$. Specifically, in the half-duplex-based F-DSTC scheme, U1 and U2 always forward $-\hat{s}_{2}^{*}$ and $\hat{s}_{1}^{*}$ to the destination in the third time frame regardless of whether $s_{2}$ and $s_{1}$ are decoded correctly or not. Hence, considering the half-duplex-based F-DSTC scheme, we can express the received signals at the destination during the three consecutive time frames of block $k$ as

$$
\left[\begin{array}{l}
y_{d}^{1} \\
y_{d}^{2} \\
y_{d}^{3}
\end{array}\right]=\sqrt{\frac{3 E_{s}}{4}}\left[\begin{array}{c}
h_{1 d} s_{1} \\
h_{2 d} s_{2} \\
-h_{1 d} \hat{s}_{2}^{*}+h_{2 d} \hat{s}_{1}^{*}
\end{array}\right]+\left[\begin{array}{c}
n_{d}^{1} \\
n_{d}^{2} \\
n_{d}^{3}
\end{array}\right]
$$

from which we can obtain

$$
\begin{aligned}
{\left[\begin{array}{c}
y_{d}^{1}+y_{d}^{2} \\
\left(y_{d}^{3}\right)^{*}
\end{array}\right] } & =\sqrt{\frac{3 E_{s}}{4}}\left[\begin{array}{cc}
h_{1 d} & h_{2 d} \\
h_{2 d}^{*} & -h_{1 d}^{*}
\end{array}\right]\left[\begin{array}{l}
s_{1} \\
s_{2}
\end{array}\right] \\
+ & {\left[\begin{array}{c}
n_{d}^{1}+n_{d}^{2} \\
h_{2 d}^{*}\left(\hat{s}_{1}-s_{1}\right)-h_{1 d}^{*}\left(\hat{s}_{2}-s_{2}\right)+\left(n_{d}^{2}\right)^{*}
\end{array}\right] . }
\end{aligned}
$$

By using the Alamouti decoding, the destination attempts to decode $s_{1}$ and $s_{2}$ from (51) as follows in (52) at the bottom of 
the page. One can see from the preceding equation that either U1 or U2 or both failing to decode would result in $\hat{s}_{1} \neq s_{1}$ and/or $\hat{s}_{2} \neq s_{2}$, causing interference at the destination in decoding both $s_{1}$ and $s_{2}$. Similar to (45), an outage probability of the half-duplex-based F-DSTC scheme can be calculate from (52) as shown in (53) at the bottom of the page, where $I_{21}$ and $I_{12}$ are given by (17) and (18), respectively. Although obtaining a closed-form outage expression for the half-duplexbased F-DSTC scheme is challenging, we can easily obtain numerical outage probabilities using (53).

3) Numerical Results: We present an outage probability comparison among the noncooperative, the half-duplex-based F-DSTC and O-DSTC schemes. Fig. 6 shows the outage probability versus transmit SNR of the noncooperative, the half-duplex-based F-DSTC and O-DSTC schemes with $R_{1}=R_{2}=1 \mathrm{bit} / \mathrm{s} / \mathrm{Hz}$. As shown in low $\mathrm{SNR}$ region, the half-duplex-based F-DSTC and O-DSTC perform worse than the noncooperative scheme in terms of the outage probability. This is due to the half-duplex relay constraint, which results in certain spectrum utilization loss and degrades the outage performance. However, in higher SNR region, the benefits achieved from diversity gain overtake the costs due to half-duplex relaying and the half-duplex-based O-DSTC scheme performs better than the noncooperative scheme. One can also observe in high SNR region of Fig. 6 that, as transmit SNR increases, the outage probability of the proposed O-DSTC scheme decreases at much higher speed than both the F-DSTC and noncooperative schemes.

In Fig. 7, we depict the outage probability versus transmit SNR $\gamma$ of the noncooperative and the half-duplex-based O-DSTC schemes with $R_{1}=r_{1} \log _{2}(1+\gamma)$ and

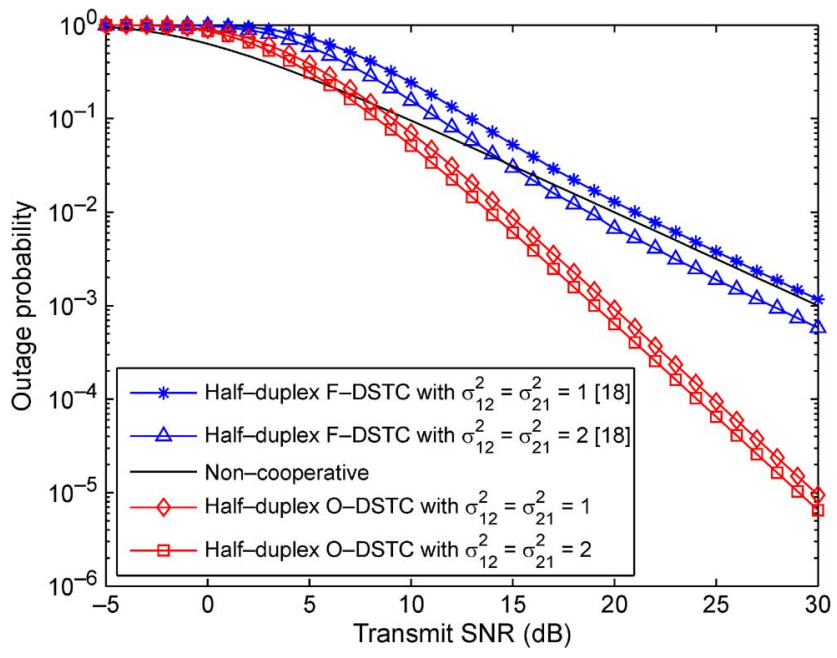

Fig. 6. Outage probability versus transmit SNR $(\gamma)$ of the noncooperative, the half-duplex-based F-DSTC and O-DSTC schemes with $R_{1}=R_{2}=1 \mathrm{bit} / \mathrm{s} / \mathrm{Hz}$ and $\sigma_{1 d}^{2}=\sigma_{2 d}^{2}=1$.

$R_{2}=r_{2} \log _{2}(1+\gamma)$, where $r_{1}$ and $r_{2}$ are called multiplexing gains of $U 1$ and $U 2$, respectively. Notice that, in the half-duplex-based F-DSTC and O-DSTC schemes, the multiplexing gains $r_{1}$ and $r_{2}$ typically vary from zero to two-third, which will be illustrated from the DMT analysis as conducted in Section IV. As shown in Fig. 7, for either $r_{1}=0.4$ or 0.3 , the outage performance of the half-duplex-based O-DSTC scheme with $r_{2}=\frac{2}{3}$ is the same as that with $r_{2}=0$. Meanwhile, as $r_{1}$ decreases from $r_{1}=0.4$ to 0.3 , the proposed O-DSTC scheme improves significantly in terms of diversity gain, as shown in Fig. 7. This implies that the diversity gain of U1 only depends

$$
\begin{array}{r}
{\left[\begin{array}{cc}
h_{1 d}^{*} & h_{2 d} \\
h_{2 d}^{*} & -h_{1 d}
\end{array}\right]\left[\begin{array}{c}
y_{d}^{1}+y_{d}^{2} \\
\left(y_{d}^{3}\right)^{*}
\end{array}\right]=\sqrt{\frac{3 E_{s}}{4}}\left[\begin{array}{cc}
\left|h_{1 d}\right|^{2}+\left|h_{2 d}\right|^{2} & 0 \\
0 & \left|h_{1 d}\right|^{2}+\left|h_{2 d}\right|^{2}
\end{array}\right]\left[\begin{array}{c}
s_{1} \\
s_{2}
\end{array}\right]} \\
+\left[\begin{array}{c}
\left|h_{2 d}\right|^{2}\left(\hat{s}_{1}-s_{1}\right)-h_{1 d}^{*} h_{2 d}\left(\hat{s}_{2}-s_{2}\right)+h_{1 d}^{*}\left(n_{d}^{1}+n_{d}^{2}\right)+h_{2 d}\left(n_{d}^{3}\right)^{*} \\
-h_{1 d} h_{2 d}^{*}\left(\hat{s}_{1}-s_{1}\right)+\left|h_{1 d}\right|^{2}\left(\hat{s}_{2}-s_{2}\right)+h_{2 d}^{*}\left(n_{d}^{1}+n_{d}^{2}\right)-h_{1 d}\left(n_{d}^{3}\right)^{*}
\end{array}\right]
\end{array}
$$

$$
\begin{aligned}
& \text { Pout }_{\text {half }- \text { FDSTC }} \\
& =\operatorname{Pr}\left(I_{12}>\frac{3 R_{1}}{2}, I_{21}>\frac{3 R_{2}}{2}\right) \operatorname{Pr}\left[\log _{2}\left(1+\frac{3\left(\left|h_{1 d}\right|^{2}+\left|h_{2 d}\right|^{2}\right)^{2} \gamma}{8\left|h_{1 d}\right|^{2}+4\left|h_{2 d}\right|^{2}}\right)<\frac{3 R_{1}}{2}\right] \\
& +\operatorname{Pr}\left(I_{12}<\frac{3 R_{1}}{2}, I_{21}>\frac{3 R_{2}}{2}\right) \operatorname{Pr}\left[\log _{2}\left(1+\frac{3\left(\left|h_{1 d}\right|^{2}+\left|h_{2 d}\right|^{2}\right)^{2} \gamma}{6\left|h_{2 d}\right|^{4} \gamma+8\left|h_{1 d}\right|^{2}+4\left|h_{2 d}\right|^{2}}\right)<\frac{3 R_{1}}{2}\right] \\
& +\operatorname{Pr}\left(I_{12}>\frac{3 R_{1}}{2}, I_{21}<\frac{3 R_{2}}{2}\right) \operatorname{Pr}\left[\log _{2}\left(1+\frac{3\left(\left|h_{1 d}\right|^{2}+\left|h_{2 d}\right|^{2}\right)^{2} \gamma}{6\left|h_{1 d}\right|^{2}\left|h_{2 d}\right|^{2} \gamma+8\left|h_{1 d}\right|^{2}+4\left|h_{2 d}\right|^{2}}\right)<\frac{3 R_{1}}{2}\right] \\
& +\operatorname{Pr}\left(I_{12}<\frac{3 R_{1}}{2}, I_{21}<\frac{3 R_{2}}{2}\right) \operatorname{Pr}\left[\log _{2}\left(1+\frac{3\left(\left|h_{1 d}\right|^{2}+\left|h_{2 d}\right|^{2}\right)^{2} \gamma}{6\left(\left|h_{2 d}\right|^{4}+\left|h_{1 d}\right|^{2}\left|h_{2 d}\right|^{2}\right) \gamma+8\left|h_{1 d}\right|^{2}+4\left|h_{2 d}\right|^{2}}\right)<\frac{3 R_{1}}{2}\right]
\end{aligned}
$$




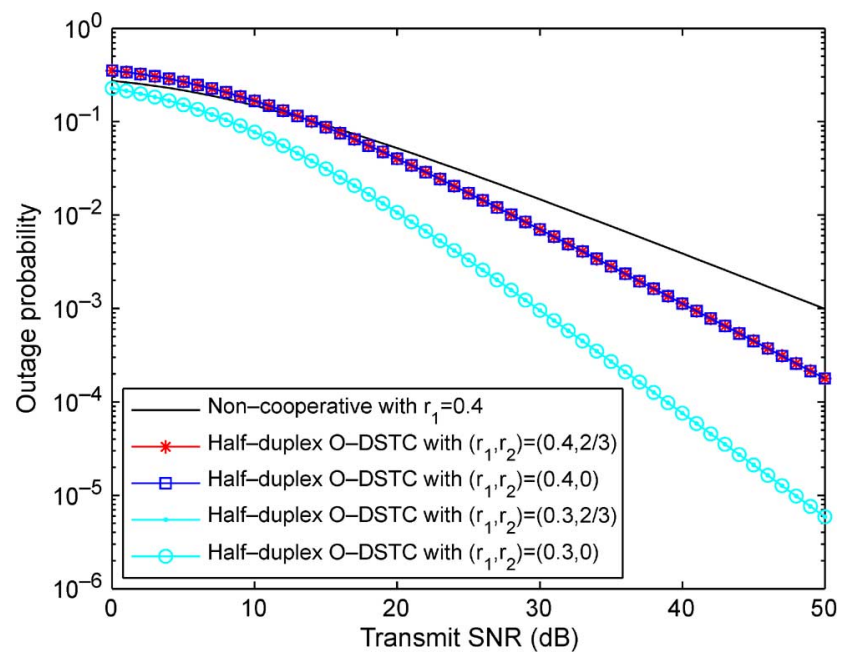

Fig. 7. Outage probability versus transmit $\operatorname{SNR}(\gamma)$ of the noncooperative and the half-duplex-based O-DSTC schemes for different multiplexing gains of U1 and $\mathrm{U} 2\left(r_{1}\right.$ and $\left.r_{2}\right)$ with $\sigma_{12}^{2}=\sigma_{1 d}^{2}=\sigma_{21}^{2}=\sigma_{2 d}^{2}=1$.

on its own multiplexing gain $\left(r_{1}\right)$ and is independent of the cooperative partner's multiplexing gain $\left(r_{2}\right)$.

\section{Conventional S-DF Cooperation}

We now study an outage probability analysis of the conventional S-DF cooperation [4] for a performance comparison with the proposed full-duplex and half-duplex-based O-DSTC schemes. According to (34)(36), an outage probability of the S-DF cooperation can be given by

$$
\begin{gathered}
\text { Pout }_{\mathrm{S}-\mathrm{DF}}=\operatorname{Pr}\left(I_{12}>2 R_{1}\right) \operatorname{Pr}\left[I_{1 d}(\varpi=1)<2 R_{1}\right] \\
+\left[1-\operatorname{Pr}\left(I_{12}>2 R_{1}\right)\right] \operatorname{Pr}\left[I_{1 d}(\varpi=2)<2 R_{1}\right]
\end{gathered}
$$

where $I_{12}, I_{1 d}(\varpi=1)$, and $I_{1 d}(\varpi=2)$ are given by (34), (35), and (36), respectively. From (34) and (36), we can easily obtain $\operatorname{Pr}\left(I_{12}>2 R_{1}\right)=\exp \left(-\frac{2^{2 R_{1}}-1}{\gamma \sigma_{12}^{2}}\right)$ and $\operatorname{Pr}\left[I_{1 d}(\varpi=\right.$ $\left.2)<2 R_{1}\right]=1-\exp \left(-\frac{2^{2 R_{1}}-1}{\gamma \sigma_{1 d}^{2}}\right)$. In addition, using (35), term $\operatorname{Pr}\left[I_{1 d}(\varpi=1)<2 R_{1}\right]$ is calculated as

$$
\begin{aligned}
\operatorname{Pr} & {\left[I_{1 d}(\varpi=1)<2 R_{1}\right] } \\
= & \operatorname{Pr}\left[\left|h_{1 d}\right|^{2}+\left|h_{2 d}\right|^{2}<\frac{2^{2 R_{1}}-1}{\gamma}\right] \\
= & \left\{\begin{array}{cc}
1-\left(1+\frac{2^{2 R_{1}}-1}{\sigma_{1 d}^{2} \gamma}\right) & \sigma_{1 d}^{2}=\sigma_{2 d}^{2} \\
\times \exp \left(-\frac{2^{2 R_{1}}-1}{\sigma_{1 d}^{2} \gamma}\right), & \\
1-\frac{\sigma_{1 d}^{2}}{\sigma_{1 d}^{2}-\sigma_{2 d}^{2}} \exp \left(-\frac{2^{2 R_{1}}-1}{\sigma_{1 d}^{2} \gamma}\right) & \\
-\frac{\sigma_{2 d}^{2}}{\sigma_{2 d}^{2}-\sigma_{1 d}^{2}} \exp \left(-\frac{2^{2 R_{1}}-1}{\sigma_{2 d}^{2} \gamma}\right), & \text { otherwise. }
\end{array}\right.
\end{aligned}
$$

So far, we have derived a closed-form outage expression for the conventional S-DF cooperation over Rayleigh fading channels. In the following, we present an outage probability comparison among the noncooperative, conventional S-DF [4], and the proposed full-duplex and half-duplex-based O-DSTC schemes.

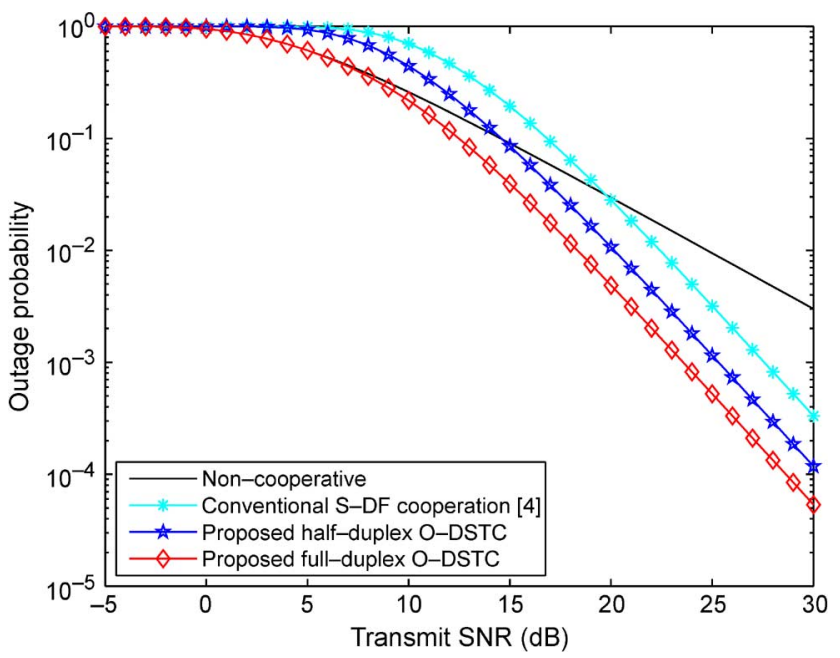

Fig. 8. Outage probability versus transmit $\operatorname{SNR}(\gamma)$ of the noncooperative, conventional S-DF cooperation [4], and the proposed full-duplex and half-duplex-based O-DSTC schemes with $R_{1}=R_{2}=2 \mathrm{bits} / \mathrm{s} / \mathrm{Hz}$ and $\sigma_{12}^{2}=$ $\sigma_{1 d}^{2}=\sigma_{21}^{2}=\sigma_{2 d}^{2}=1$.

Fig. 8 shows the outage probability versus transmit SNR $\gamma$ of the noncooperation, conventional S-DF cooperation [4] and the proposed full-duplex and half-duplex-based O-DSTC schemes. One can observe from Fig. 8 that the full-duplex and half-duplex-based O-DSTC schemes outperform the conventional S-DF cooperation by about 5 and $3 \mathrm{~dB}$, respectively. This indirectly shows the advantage of proposed O-DSTC over the superposition modulation based cooperative diversity [7], [8], since the latter approach outperforms the conventional S-DF cooperation by $1.5-2 \mathrm{~dB}$ only as reported in [7] and [8].

\section{Diversity-MultipleXing TradeOfF ANAlysis}

In this section, we conduct the DMT analysis for the proposed full-duplex and half-duplex-based O-DSTC schemes. Following [22], the diversity gain of a wireless transmission system can be defined as

$$
d=-\lim _{\gamma \rightarrow \infty} \frac{\log [\operatorname{Pout}(\gamma)]}{\log (\gamma)}
$$

where $\operatorname{Pout}(\gamma)$ represents an outage probability of the wireless transmission system and $\gamma$ is the transmit SNR. Meanwhile, given multiplexing gains $r_{1}$ and $r_{2}$, the date rates of $\mathrm{U} 1$ and $\mathrm{U} 2$ (i.e., $R_{1}$ and $R_{2}$ ) are given by

$$
R_{1}=r_{1} \log _{2}(1+\gamma)
$$

and

$$
R_{2}=r_{2} \log _{2}(1+\gamma) .
$$

By using (37), (56), and (57), the DMT of the noncooperative scheme is easily obtained as

$$
d_{\text {non }}+r_{1}=1
$$


where $0 \leq r_{1} \leq 1$. One can see from (59) that a maximum diversity gain $d_{\text {non }}=1$ is achieved as $r_{1} \rightarrow 0$ and, on the other hand, a maximum multiplexing gain of one is obtained as $d_{\text {non }} \rightarrow 0$. In the following, we examine the DMT for the fullduplex and half-duplex-based O-DSTC and F-DSTC schemes.

\section{A. Full-Duplex-Based O-DSTC Scheme}

Considering $\gamma \rightarrow \infty$ and following (38), we calculate an outage probability limit of the full-duplex-based O-DSTC scheme as

$$
\begin{aligned}
\lim _{\gamma \rightarrow \infty} & \text { Pout }_{\text {full-ODSTC }} \\
= & \lim _{\gamma \rightarrow \infty} \operatorname{Pr}\left(I_{12}>R_{1}, I_{21}>R_{2}\right) \lim _{\gamma \rightarrow \infty} \operatorname{Pr}\left[I_{1 d}(\theta=1)<R_{1}\right] \\
& +\left[1-\lim _{\gamma \rightarrow \infty} \operatorname{Pr}\left(I_{12}>R_{1}, I_{21}>R_{2}\right)\right] \\
& \times \lim _{\gamma \rightarrow \infty} \operatorname{Pr}\left[I_{1 d}(\theta=2)<R_{1}\right]
\end{aligned}
$$

From (39), we can easily obtain $\lim _{\gamma \rightarrow \infty} \operatorname{Pr}\left(I_{12}>R_{1}, I_{21}>\right.$ $\left.R_{2}\right)=1$. Using the result of Appendix A, we have

$\lim _{\gamma \rightarrow \infty} \operatorname{Pr}\left[I_{1 d}(\theta=1)<R_{1}\right]=\frac{2}{\sigma_{1 d}^{2} \sigma_{2 d}^{2}} \cdot \frac{\left(2^{R_{1}}-1\right)^{2}}{\gamma^{2}}+O\left(\frac{1}{\gamma^{2}}\right)$

where $O(\cdot)$ represents the higher-order terms. In addition, by considering $\gamma \rightarrow \infty$ and using Taylor series expansion, term $\lim _{\gamma \rightarrow \infty}\left[1-\operatorname{Pr}\left(I_{12}>R_{1}, I_{21}>R_{2}\right)\right]$ can be expanded as

$$
\begin{aligned}
& \lim _{\gamma \rightarrow \infty}\left[1-\operatorname{Pr}\left(I_{12}>R_{1}, I_{21}>R_{2}\right)\right] \\
& =\lim _{\gamma \rightarrow \infty}\left[1-\exp \left(-\frac{2\left(2^{R_{1}}-1\right)}{\gamma \sigma_{12}^{2}}\right) \exp \left(-\frac{2\left(2^{R_{2}}-1\right)}{\gamma \sigma_{21}^{2}}\right)\right] \\
& =\frac{2}{\sigma_{12}^{2}} \cdot \frac{2^{R_{1}}-1}{\gamma}+\frac{2}{\sigma_{21}^{2}} \cdot \frac{2^{R_{2}}-1}{\gamma}+O\left(\frac{1}{\gamma}\right) .
\end{aligned}
$$

Similarly, applying Taylor approximation to (41), we have

$$
\lim _{\gamma \rightarrow \infty} \operatorname{Pr}\left[I_{1 d}(\theta=2)<R_{1}\right]=\frac{1}{\sigma_{1 d}^{2}} \cdot \frac{2^{R_{1}}-1}{\gamma}+O\left(\frac{1}{\gamma}\right) .
$$

By substituting (61)-(63) into (60) and combining these results with (56)-(58), the DMT of the full-duplex-based O-DSTC scheme is obtained as

$$
d_{\text {full-ODSTC }}+r_{1}+\max \left(r_{1}, r_{2}\right)=2, \quad 0 \leq\left(r_{1}, r_{2}\right) \leq 1
$$

which shows that a maximum diversity gain of two is obtained as $\left(r_{1}, r_{2}\right) \rightarrow 0$. One can observe from (64) that the diversity gain of U1 not only depends on its own multiplexing gain $r_{1}$, but also relates to its partner's multiplexing gain $r_{2}$. This reason is that, either $\mathrm{U} 1$ or $\mathrm{U} 2$ increases the multiplexing gain (i.e., higher data rate), it decreases the probability of occurrence of case $\theta=$ 1 and increases the occurrence probability of the other case $\theta=$ 2. Moreover, under cases $\theta=1$ and 2, different diversity gains are achieved by $\mathrm{U} 1$ as implied from (8) and (11), which finally leads to the fact that the diversity gain of U1 depends on both $r_{1}$ and $r_{2}$. From (64), given a U1's multiplexing gain $r_{1}$, the diversity gain of the U1's transmissions can be maximized when $r_{2} \leq r_{1}$. Also, one can imagine that given $r_{2}$, the diversity gain

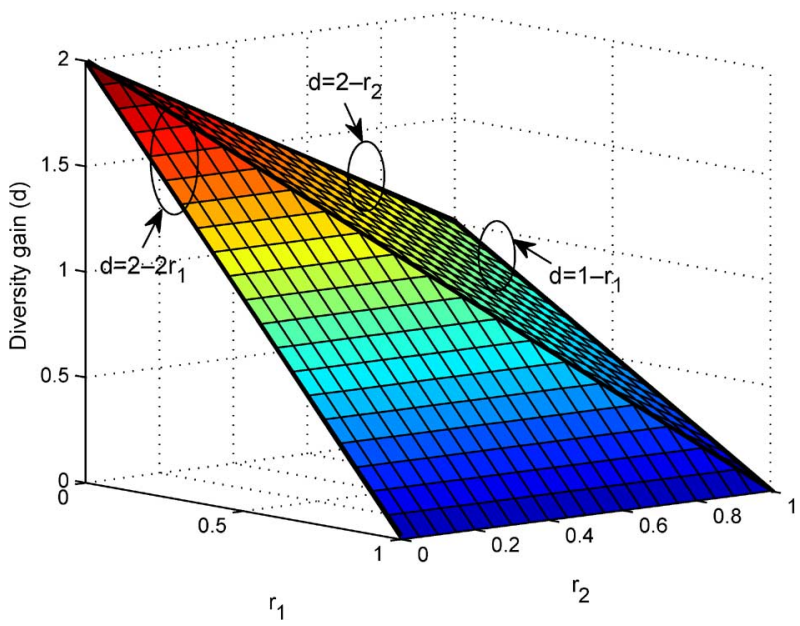

Fig. 9. Diversity gain of the full-duplex-based O-DSTC scheme versus multiplexing gains of $\mathrm{U} 1$ and $\mathrm{U} 2\left(r_{1}\right.$ and $\left.r_{2}\right)$.

of the U2's transmissions is given by $2-r_{2}-\max \left(r_{1}, r_{2}\right)$, which is maximized with $r_{1} \leq r_{2}$. Therefore, by jointly considering $\mathrm{U} 1$ and $\mathrm{U} 2$, an optimal DMT of the proposed full-duplex-based O-DSTC scheme is achieved when $r_{1}=r_{2}$.

In addition, one can see from (64) that given $r_{1}=r_{2}$, the DMT of the full-duplex-based O-DSTC scheme is $d_{\text {full-ODSTC }}=2\left(1-r_{1}\right)$, which indicates that the maximum multiplexing gain is one. This is an optimal DMT for the $2 \times 1$ multiple-input and single-output (MISO) channel, as discussed in [22]. Moreover, it is proven in [22] that the maximum multiplexing gain of multiple-input and multiple-output (MIMO) channel is shown as the minimum of the number of transmit antennas and that of receiver antennas. Notice that the two cooperative users (i.e., U1 and U2) and one common destination can form a distributed $2 \times 1$ MISO channel. Therefore, it is feasible to achieve a multiplexing gain higher than one by increasing the number of receive antennas at the common destination.

In Fig. 9, we plot the diversity gain of the U1's transmissions as a function of multiplexing gains $r_{1}$ and $r_{2}$ using (64). One can easily observe from Fig. 9 that given $r_{1}$, a maximum diversity gain, i.e., $2-2 r_{1}$, is achieved when $r_{2} \leq r_{1}$. As shown in Fig. 9, for $r_{2}=1$, the diversity gain of U1's transmissions by using the full-duplex-based O-DSTC is $1-r_{1}$, which is the same as that of the noncooperative scheme as given by (59). It is pointed out that, considering the full-duplex-based O-DSTC and $r_{1}=1$, the diversity gain of U2's transmissions can similarly be obtained as $1-r_{2}$. Therefore, for the full-duplex-based O-DSTC scheme, one can conclude that, when U1 (or U2) transmits its own information at the full rate, the DMT performance of its partner is degraded to be the same as that of the noncooperative scheme.

For the purpose of comparison, let us examine the DMT of the full-duplex-based F-DSTC scheme [18]. Letting $\gamma \rightarrow \infty$ and following (4) and (5), we easily obtain that $\lim _{\gamma \rightarrow \infty} \operatorname{Pr}\left(I_{12}>R_{1}, I_{21}>R_{2}\right)$ is equal to one, and terms $\lim _{\gamma \rightarrow \infty} \operatorname{Pr}\left(I_{12}<R_{1}, I_{21}>R_{2}\right), \lim _{\gamma \rightarrow \infty} \operatorname{Pr}\left(I_{12}>R_{1}, I_{21}<R_{2}\right)$ 
and $\lim _{\gamma \rightarrow \infty} \operatorname{Pr}\left(I_{12}<R_{1}, I_{21}<R_{2}\right)$ behave as $\frac{2^{R_{1}}-1}{\gamma}$, $\frac{2^{R_{2}}-1}{\gamma}$ and $\frac{\left(2^{R_{1}}-1\right)\left(2^{R_{2}}-1\right)}{\gamma^{2}}$, respectively. Similar to (61), $\lim _{\gamma \rightarrow \infty} \operatorname{Pr}\left[\log _{2}\left(1+\frac{\left(\left|h_{1 d}\right|^{2}+\left|h_{2 d}\right|^{2}\right) \gamma}{2}\right)<R_{1}\right] \quad$ behaves as $\frac{\left(2^{R_{1}}-1\right)^{2}}{\gamma^{2}}$. In addition, one can see that terms

$$
\begin{aligned}
& \lim _{\gamma \rightarrow \infty} \operatorname{Pr}\left[\log _{2}\left(1+\frac{\left(\left|h_{1 d}\right|^{2}+\left|h_{2 d}\right|^{2}\right)^{2}}{2\left|h_{2 d}\right|^{4}}\right)<R_{1}\right], \\
& \lim _{\gamma \rightarrow \infty} \operatorname{Pr}\left[\log _{2}\left(1+\frac{\left(\left|h_{1 d}\right|^{2}+\left|h_{2 d}\right|^{2}\right)^{2}}{2\left|h_{2 d}\right|^{4}}\right)<R_{1}\right],
\end{aligned}
$$

and

$$
\lim _{\gamma \rightarrow \infty} \operatorname{Pr}\left[\log _{2}\left(1+\frac{\left(\left|h_{1 d}\right|^{2}+\left|h_{2 d}\right|^{2}\right)^{2}}{2\left(\left|h_{2 d}\right|^{4}+\left|h_{1 d}\right|^{2}\left|h_{2 d}\right|^{2}\right)}\right)<R_{1}\right],
$$

respectively, converge to nonzero constants. Substituting these results into (45) and combining with (56)-(58), we can easily obtain the DMT of the full-duplex-based F-DSTC scheme as

$$
d_{\text {full-FDSTC }}+\max \left(r_{1}, r_{2}\right)=1, \quad 0 \leq\left(r_{1}, r_{2}\right) \leq 1
$$

which shows that a maximum diversity gain of only one is achieved as $\left(r_{1}, r_{2}\right) \rightarrow 0$. This is due to the fact that, in the full-duplex-based F-DSTC scheme, a failure in decoding the partner's information at any of the two users (i.e., U1 and U2) results in interference at the destination in decoding both users' information, leading to a maximum diversity gain of only one. In addition, one can see from (65) that, when either U1 or U2 transmits its own information at the full rate, the diversity gain of the full-duplex-based F-DSTC becomes zero. This is because that either $\mathrm{U} 1$ or $\mathrm{U} 2$ with the full rate transmission results in its partner always failing to decode its information, which always leads to interference at the destination in decoding both users' information.

\section{B. Half-Duplex-Based O-DSTC Scheme}

We investigate the DMT performance of the half-duplex-based O-DSTC scheme. Letting $\gamma \rightarrow \infty$ and following (19), we can obtain

$\lim _{\gamma \rightarrow \infty} \operatorname{Pr}(\omega=1)=1$,

$\lim _{\gamma \rightarrow \infty} \operatorname{Pr}(\omega=2)=\frac{4}{3 \sigma_{12}^{2}} \cdot \frac{2^{\frac{3 R_{1}}{2}}-1}{\gamma}+O\left(\frac{1}{\gamma}\right)$,

$\lim _{\gamma \rightarrow \infty} \operatorname{Pr}(\omega=3)=\frac{4}{3 \sigma_{21}^{2}} \cdot \frac{2^{\frac{3 R_{2}}{2}}-1}{\gamma}+O\left(\frac{1}{\gamma}\right)$

and

$\lim _{\gamma \rightarrow \infty} \operatorname{Pr}(\omega=4)=\frac{16}{9 \sigma_{12}^{2} \sigma_{21}^{2}} \cdot \frac{\left(2^{\frac{3 R_{1}}{2}}-1\right)\left(2^{\frac{3 R_{2}}{2}}-1\right)}{\gamma^{2}}+O\left(\frac{1}{\gamma^{2}}\right)$

In addition, following (48), we have $\lim _{\gamma \rightarrow \infty} \operatorname{Pr}\left[I_{1 d}(\omega=1)<\frac{3 R_{1}}{2}\right]=\lim _{\gamma \rightarrow \infty} \operatorname{Pr}\left[I_{1 d}(\omega=3)<\frac{3 R_{1}}{2}\right]=$ $\frac{32}{9 \sigma_{1 d}^{2} \sigma_{2 d}^{2}} \cdot \frac{\left(2^{\frac{3 R_{1}}{2}}-1\right)^{2}}{\gamma^{2}}+O\left(\frac{1}{\gamma^{2}}\right)$. Similarly, from (49), we obtain $\lim _{\gamma \rightarrow \infty} \operatorname{Pr}\left[I_{1 d}(\omega=2)<\frac{3 R_{1}}{2}\right]=\lim _{\gamma \rightarrow \infty} \operatorname{Pr}\left[I_{1 d}(\omega=4)<\frac{3 R_{1}}{2}\right]=$ $\frac{4}{3 \sigma_{1 d}^{2}} \cdot \frac{2^{\frac{3 R_{1}}{2}}-1}{\gamma}+O\left(\frac{1}{\gamma}\right)$. Substituting these results into and combining with Eqs. (56)-(58), we can obtain the DMT of the half-duplex-based O-DSTC scheme as

$$
d_{\text {half-ODSTC }}+3 r_{1}=2, \quad 0 \leq r_{1} \leq 2 / 3
$$

which shows that a maximum diversity gain of two is obtained as the multiplexing gain $r_{1}$ approaches zero. One can also observe from (66) that the diversity gain achieved by U1 only depends on its own multiplexing gain $r_{1}$ and has nothing to do with its partner's multiplexing gain $r_{2}$. This is because that the U2's multiplexing gain only affects U1 in decoding U2's information. However, no matter whether U1 succeeds in decoding U2's information or not, the diversity gain of U1 keeps unchanged, e.g., $I_{1 d}(\omega=1)=I_{1 d}(\omega=3)$ and $I_{1 d}(\omega=$ $2)=I_{1 d}(\omega=4)$, as implied from (22), (30), (26) and (32). One can also observe from these equations that the U1's diversity gain only relates to whether U2 succeeds in decoding U1's information, which is irrelevant to the U2's multiplexing gain. Therefore, the DMT of U1 and U2 are independent of each other in the half-duplex-based O-DSTC scheme, differing from the full-duplex-based O-DSTC scheme where mutual dependence exists between U1 and U2 in terms of DMT, as shown in (64). We now examine the DMT of the half-duplex-based F-DSTC scheme [18]. By considering $\gamma \rightarrow \infty$, it is easy from (53) to ob-

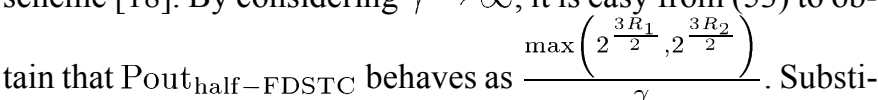
tuting this result as well as (57) and (58) into ${ }^{\gamma}(56)$ gives

$$
d_{\text {half-FDSTC }}+\frac{3}{2} \max \left(r_{1}, r_{2}\right)=1
$$

where $0 \leq r_{1} \leq \frac{2}{3}$ and $0 \leq r_{2} \leq \frac{2}{3}$. As shown in (67), a maximum diversity gain of only one is achieved as $\left(r_{1}, r_{2}\right) \rightarrow 0$. Moreover, mutual dependence between U1 and U2 exists in terms of DMT performance, which arises from the fact that either U1 or U2 failing to decode would result in interference at the destination in decoding both users' information, as shown in (52).

Fig. 10 shows a DMT comparison among the noncooperative, the conventional S-DF cooperation [4], the half-duplex-based F-DSTC and O-DSTC, and the full-duplex-based F-DSTC and O-DSTC schemes with $r_{1}=r_{2}$. Notice that the DMT curve of the noncooperative scheme is identical to that of the full-duplex-based F-DSTC scheme. As shown in Fig. 10, as the multiplexing gains $r_{1}$ and $r_{2}$ approach zero, both the full-duplex and the half-duplex-based F-DSTC schemes [18] achieve a diversity gain of one. However, no matter which duplex mode is adopted, a maximum diversity gain of two is obtained by the proposed O-DSTC scheme, showing its advantage over the F-DSTC scheme. One can also observe from Fig. 10 that both the full-duplex and half-duplex-based O-DSTC schemes strictly outperform the conventional S-DF cooperation [4] in terms of the DMT performance.

\section{CONCLUSiON}

In this paper, we explored O-DSTC for DF cooperation systems. We proposed the full-duplex and half-duplex-based O-DSTC schemes and evaluated their outage probability performance over Rayleigh fading channels. For the comparison 


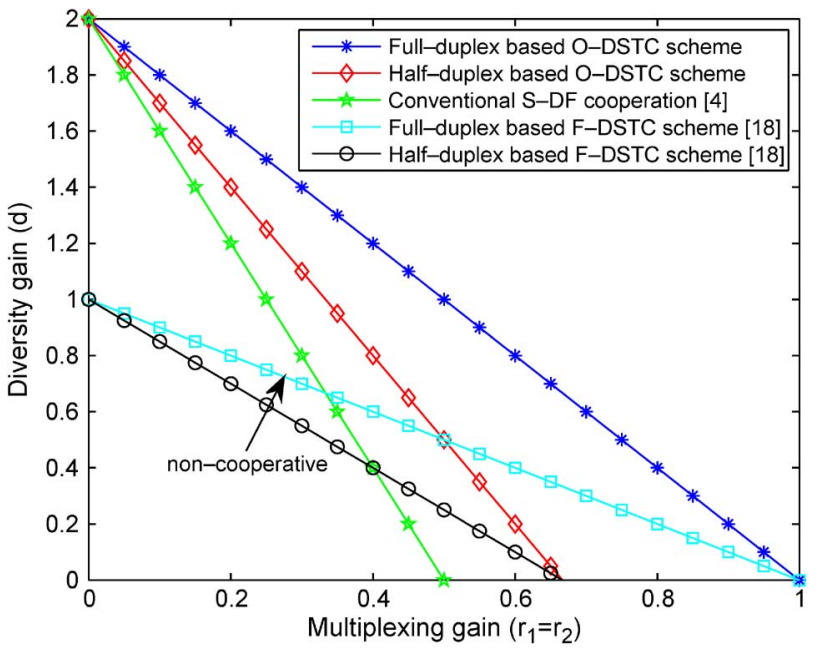

Fig. 10. Diversity-multiplexing tradeoffs of the noncooperative, the conventional S-DF cooperation [4], the half-duplex-based F-DSTC and O-DSTC, and the full-duplex-based F-DSTC and O-DSTC schemes with $r_{1}=r_{2}$.

purpose, we conducted an outage analysis for the noncooperative, the conventional S-DF cooperation, and the full-duplex and half-duplex-based fixed DSTC (F-DSTC) schemes. Numerical results showed that the proposed O-DSTC scheme outperforms the conventional S-DF and F-DSTC schemes in terms of the outage probability considering both the full-duplex and half-duplex modes. In addition, we examined the DMT of the full-duplex and half-duplex-based O-DSTC and F-DSTC schemes as well as the conventional S-DF cooperation. It was shown that, no matter which duplex mode (i.e., full-duplex and half-duplex) is considered, the proposed O-DSTC scheme strictly outperforms the conventional S-DF and F-DSTC schemes. We also illustrated that, in the full-duplex-based O-DSTC scheme, mutual dependence exits between two cooperative users in terms of DMT. However, for the half-duplex-based O-DSTC scheme, the DMT performance of the two users are independent of each other, i.e., the diversity gain of a user only relates to its own multiplexing gain.

\section{APPENDIX A}

PROOF OF EQ. (61)

Using (8) and letting $x=\left|h_{1 d}\right|^{2}, y=\left|h_{2 d}\right|^{2}$ and $\Delta=\frac{2\left(2^{R_{1}}-1\right)}{\gamma}$, we can rewrite $\lim _{\gamma \rightarrow \infty} \operatorname{Pr}\left[I_{1 d}(\theta=1)<R\right]$ as $\lim _{\Delta} \operatorname{Pr}[x+y<\Delta]$. Notice that random variables $x \stackrel{\Delta \rightarrow 0}{=}\left|h_{1 d}\right|^{2}$ and $y=\left|h_{2 d}\right|^{2}$ follow exponential distributions with means $\sigma_{1 d}^{2}$ and $\sigma_{2 d}^{2}$, respectively. Thus, we can calculate $\lim _{\Delta \rightarrow 0} \operatorname{Pr}[x+y<\Delta]$ as

$$
\begin{aligned}
& \lim _{\Delta \rightarrow 0} \operatorname{Pr}[x+y<\Delta] \\
& =\lim _{\Delta \rightarrow 0} \int_{0}^{\Delta} \frac{1}{\sigma_{1 d}^{2}} \exp \left(-\frac{x}{\sigma_{1 d}^{2}}\right) d x \int_{0}^{\Delta-x} \frac{1}{\sigma_{2 d}^{2}} \exp \left(-\frac{y}{\sigma_{2 d}^{2}}\right) d y \\
& =\lim _{\Delta \rightarrow 0} \int_{0}^{\Delta} \frac{1}{\sigma_{1 d}^{2}} \exp \left(-\frac{x}{\sigma_{1 d}^{2}}\right)\left[1-\exp \left(-\frac{\Delta-x}{\sigma_{2 d}^{2}}\right)\right] d x
\end{aligned}
$$

which shows $0<x<\Delta$. Considering $0<x<\Delta$ and letting $\Delta \rightarrow 0$, we can obtain from Taylor series

$$
1-\exp \left(-\frac{\Delta-x}{\sigma_{2 d}^{2}}\right)=\frac{\Delta-x}{\sigma_{2 d}^{2}}+O\left(\frac{\Delta-x}{\sigma_{2 d}^{2}}\right) .
$$

Substituting (A.2) into (A.1) yields

$$
\begin{aligned}
\lim _{\Delta \rightarrow 0} \operatorname{Pr}[x+y<\Delta] \\
=\lim _{\Delta \rightarrow 0} \int_{0}^{\Delta} \frac{\Delta-x}{\sigma_{1 d}^{2} \sigma_{2 d}^{2}} \exp \left(-\frac{x}{\sigma_{1 d}^{2}}\right) d x \\
=\lim _{\Delta \rightarrow 0} \int_{0}^{\Delta} \frac{\Delta}{\sigma_{1 d}^{2} \sigma_{2 d}^{2}} \exp \left(-\frac{x}{\sigma_{1 d}^{2}}\right) d x \\
\quad-\lim _{\Delta \rightarrow 0} \int_{0}^{\Delta} \frac{x}{\sigma_{1 d}^{2} \sigma_{2 d}^{2}} \exp \left(-\frac{x}{\sigma_{1 d}^{2}}\right) d x \\
=\lim _{\Delta \rightarrow 0} \frac{\Delta}{\sigma_{2 d}^{2}}\left[1-\exp \left(-\frac{\Delta}{\sigma_{1 d}^{2}}\right)\right] \\
\quad+\frac{\Delta}{\sigma_{2 d}^{2}}-\frac{\sigma_{1 d}^{2}}{\sigma_{2 d}^{2}}\left[1-\exp \left(-\frac{\Delta}{\sigma_{1 d}^{2}}\right)\right] .
\end{aligned}
$$

Applying Taylor series expansion to the above equation obtains

$$
\lim _{\Delta \rightarrow 0} \operatorname{Pr}[x+y<\Delta]=\frac{\Delta^{2}}{2 \sigma_{1 d}^{2} \sigma_{2 d}^{2}}+O\left(\Delta^{2}\right) .
$$

Substituting $\Delta=\frac{2\left(2^{R_{1}}-1\right)}{\gamma}$ into (A.4) gives

$\lim _{\gamma \rightarrow \infty} \operatorname{Pr}\left[x+y<\frac{2\left(2^{R_{1}}-1\right)}{\gamma}\right]=\frac{2}{\sigma_{1 d}^{2} \sigma_{2 d}^{2}} \cdot \frac{\left(2^{R_{1}}-1\right)^{2}}{\gamma^{2}}+O\left(\frac{1}{\gamma^{2}}\right)$

This completes the proof of (61).

\section{REFERENCES}

[1] R. T. Derryberry, S. D. Gray, D. M. Lonescu, G. Mandyam, and B. Raghothaman, "Transmit diversity in 3G CDMA systems," IEEE Commun. Mag., vol. 40, no. 4, pp. 68-75, Apr. 2002.

[2] A. Nosratinia and T. E. Hunter, "Cooperative communication in wireless networks," IEEE Commun. Mag., vol. 42, no. 10, pp. 74-80, 2004.

[3] A. Sendonaris, E. Erkip, and B. Aazhang, "User cooperation diversity—Part I: System description," IEEE Trans. Commun., vol. 51, no. 11, pp. 1927-1938, 2003.

[4] J. N. Laneman, D. N. C. Tse, and G. W. Wornell, "Cooperative diversity in wireless networks: Efficient protocols and outage behavior," IEEE Trans. Inf. Theory, vol. 50, pp. 3062-3080, Dec. 2004.

[5] T. E. Hunter, S. Sanayei, and A. Nosratinia, "Outage analysis of coded cooperation," IEEE Trans. Inf. Theory, vol. 52, no. 2, pp. 375-391, Feb. 2006.

[6] K. Azarian, H. E. Gamal, and P. Schniter, "On the achievable diversity-multiplexing tradeoff in half-duplex cooperative channels," IEEE Trans. Inf. Theory, vol. 51, pp. 4152-4172, Nov. 2005.

[7] E. G. Larsson and B. R. Vojcic, "Cooperative transmit diversity based on superposition modulation," IEEE Commun. Lett., vol. 9, pp. 778-780, Sep. 2005.

[8] Z. Ding, T. Ratnarajah, and C. C. F. Cowan, "On the diversity-multiplexing tradeoff for wireless cooperative multiple access systems," IEEE Trans. Signal Process., vol. 55, no. 9, pp. 4627-4638, Sep. 2007.

[9] S. Wei, "Diversity-multiplexing tradeoff of asynchronous cooperative diversity in wireless networks," IEEE Trans. Inf. Theory, vol. 53, no. 11, pp. 4150-4172, Nov. 2007.

[10] Y. Zou, J. Zhu, B. Zheng, and Y.-D. Yao, "An adaptive cooperation diversity scheme with best-relay selection in cognitive radio networks," IEE E Trans. Signal Process., vol. 58, no. 10, pp. 5438-5445, Oct. 2010. 
[11] Y. Zou, Y.-D. Yao, and B. Zheng, "A selective-relay based cooperative spectrum sensing without dedicated reporting channels in cognitive radio networks," IEEE Trans. Wireless Commun., vol. 10, no. 4, pp. 1188-1198, Apr. 2011.

[12] Y. Zou, Y.-D. Yao, and B. Zheng, "A cooperative sensing based cognitive relay transmission scheme without a dedicated sensing relay channel in cognitive radio networks," IEEE Trans. Signal Process., vol. 59, no. 2, pp. 854-858, Feb. 2011.

[13] S. M. Alamouti, "A simple transmit diversity technique for wireless communications," IEEE J. Sel. Areas Commun., vol. 16, no. 8, pp. 1451-1458, Oct. 1998.

[14] V. Tarokh, N. Seshadri, and A. R. Calderbank, "Space-time codes for high data rate wireless communication: Performance criterion and code construction," IEEE Trans. Inf. Theory, vol. 44, pp. 744-765, 1998.

[15] B. M. Hochwald and T. L. Marzetta, "Unitary space-time modulation for multiple-antenna communication in Rayleigh flat-fading," IEEE Trans. Inf. Theory, vol. 46, pp. 543-564, Mar. 2000.

[16] J. N. Laneman and G. W. Wornell, "Distributed space-time-coded protocols for exploiting cooperative diversity in wireless networks," IEEE Trans. Inf. Theory, vol. 49, no. 10, pp. 2415-2425, Oct. 2003.

[17] Y. Jing and B. Hassibi, "Distributed space-time coding in wireless relay networks," IEEE Trans. Wireless Commun., vol. 5, no. 12, pp. 3524-3536, Dec. 2006.

[18] G. Scutari and S. Barbarossa, "Distributed space-time coding for regenerative relay networks," IEEE Trans. Wireless Commun., vol. 4, no. 5, pp. 2387-2399, Sep. 2005.

[19] J. Abouei, H. Bagheri, and A. K. Khandani, "An efficient adaptive distributed space-time coding scheme for cooperative relaying," IEEE Trans. Wireless Commun., vol. 8, no. 10, pp. 4957-4962, Oct. 2009.

[20] J. I. Choiy, M. Jainy, K. Srinivasany, P. Levis, and S. Katti, "Achieving single channel, full duplex wireless communication," in Proc. ACM MobiCom 2010, IL.

[21] A. Nosratinia and T. E. Hunter, "Grouping and partner selection in cooperative wireless networks," IEEE J. Sel. Areas Commun., vol. 25, no. 2, pp. 369-378, Feb. 2007.

[22] L. Zheng and D. Tse, "Diversity and multiplexing: A fundamental tradeoff in multiple antenna channels," IEEE Trans. Inf. Theory, vol. 49, no. 5, pp. 1073-1096, May 2003.

[23] Y. Zou, Y.-D. Yao, and B. Zheng, "Outage probability analysis of cognitive transmissions: The impact of spectrum sensing overhead," IEEE Trans. Wireless Commun., vol. 9, no. 8, pp. 2676-2688, Aug. 2010.

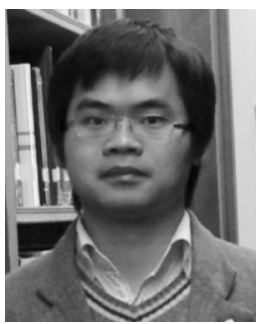

Yulong Zou (S'09-M'11) received the B.Eng. degree in Information Engineering from Nanjing University of Posts and Telecommunications (NUPT), Nanjing China, in 2006.

$\mathrm{He}$ is currently working toward the dual Ph.D. degree at the Institute of Signal Processing and Transmission of NUPT, and the Electrical and Computer Engineering Department of Stevens Institute of Technology (SIT), Hoboken, NJ. His research interests span a wide range of topics in wireless communications and signal processing, including cooperative communications, space-time coding, network coding, and cognitive radio. Recently, he has been working on the cooperative relay techniques in cognitive radio networks, opportunistic distributed space-time coding in cooperative wireless networks, and full-diversity high-rate network coding for cellular systems (e.g., LTE/IMT-advanced and beyond).

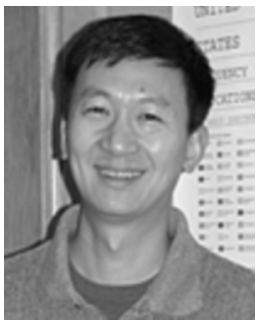

Yu-Dong Yao (S'88-M'88-SM'94-F'11) received the B.Eng. and M.Eng. degrees from the Nanjing University of Posts and Telecommunications, Nanjing, China, in 1982 and 1985, respectively, and the Ph.D. degree from Southeast University, Nanjing, in 1988, all in electrical engineering.

He has been with Stevens Institute of Technology, Hoboken, NJ, since 2000 and is currently a Professor and Department Director of Electrical and Computer Engineering. He is also a Director of Stevens' Wireless Information Systems Engineering Laboratory (WISELAB). From 1989 and 1990, he was with Carleton University, Ottawa, Canada, as a Research Associate working on mobile radio communications. From 1990 to 1994, he was with Spar Aerospace Ltd., Montreal, Canada, where he was involved in research on satellite communications. From 1994 to 2000, he was with Qualcomm Inc., San Diego, CA, where he participated in research and development in wireless code-division multiple-access (CDMA) systems. He holds one Chinese patent and 12 U.S. patents. His research interests include wireless communications and networks, spread spectrum and CDMA, antenna arrays and beamforming, cognitive and software defined radio (CSDR), and digital signal processing for wireless systems.

Dr. Yao was an Associate Editor of the IEEE COMMUNICATIONS LETTERS and IEEE TRANSACtions ON Vehicular TEChNOLOGY, and an Editor for the IEEE TRANSACTIONS ON WIRELESS COMMUNICATIONS.

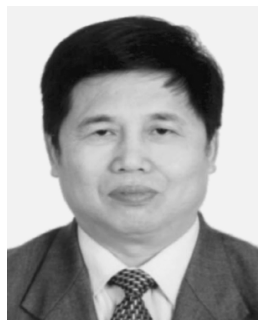

Baoyu Zheng (M'06) received the B.S. and M.S. degrees from the Department of Circuit and Signal System, Nanjing University of Posts and Telecommunications (NUPT), Nanjing, China, in 1969 and 1981, respectively.

Since then, he has been engaged in teaching and researching with the Signal and Information Processing. He is a full professor and doctoral advisor at NUPT. His research interests span the broad area of the intelligent signal processing, wireless network and signal processing for modern communication, and the quantum signal processing. 